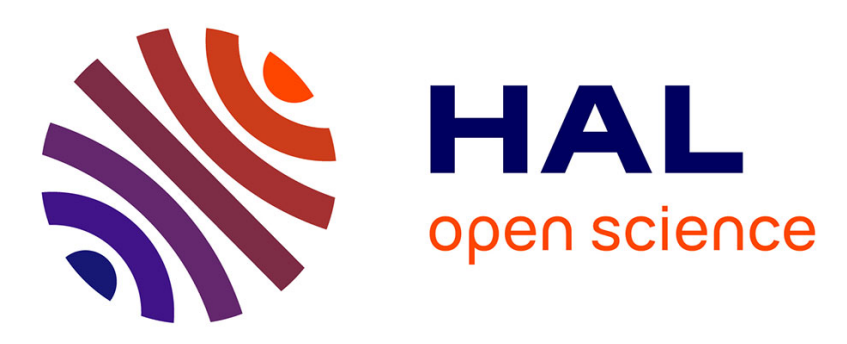

\title{
Investigations of the thermomechanical behavior of a coarse-grained aluminum multicrystal using Constrained full-field measurements methods
}

\author{
Li Li, Félix Latourte, Jean Michel Muracciole, Laurent Waltz, Laurent \\ Sabatier, Bertrand Wattrisse
}

\section{To cite this version:}

Li Li, Félix Latourte, Jean Michel Muracciole, Laurent Waltz, Laurent Sabatier, et al.. Investigations of the thermomechanical behavior of a coarse-grained aluminum multicrystal using Constrained full-field measurements methods. Optics and Lasers in Engineering, 2019, 112, pp.182-195. 10.1016/j.optlaseng.2018.08.003 . hal-01885346

\author{
HAL Id: hal-01885346 \\ https://hal.science/hal-01885346
}

Submitted on 1 Oct 2018

HAL is a multi-disciplinary open access archive for the deposit and dissemination of scientific research documents, whether they are published or not. The documents may come from teaching and research institutions in France or abroad, or from public or private research centers.
L'archive ouverte pluridisciplinaire HAL, est destinée au dépôt et à la diffusion de documents scientifiques de niveau recherche, publiés ou non, émanant des établissements d'enseignement et de recherche français ou étrangers, des laboratoires publics ou privés. 


\title{
Investigations of the thermomechanical behavior of a coarse-grained aluminum multicrystal using Constrained full-field measurements methods
}

\author{
L. Li ${ }^{\text {a, *, F. Latourte }}{ }^{\text {b }}$, J.-M. Muracciole ${ }^{\text {a, c }}$, L. Waltz ${ }^{\text {a, c }}$, L. Sabatier ${ }^{\text {a, d }}$, B. Wattrisse ${ }^{\text {a, c }}$ \\ ${ }^{a}$ LMGC, Univ. Montpellier, CNRS, Montpellier, France \\ ${ }^{\mathrm{b}}$ EDF R\&D, MMC Department, Moret-sur-Loing, France \\ ${ }^{\mathrm{c}}$ Laboratoire de Micromécanique et d'Intégrité des Structures (MIST), IRSN-CNRS-Université de Montpellier, Montpellier, France \\ ${ }^{\mathrm{d}}$ Aix Marseille Univ, CNRS, Centrale Marseille, LMA UMR 7031, Marseille, France
}

Keywords:

Digital image correlation (DIC)

Constrained DIC

InfraRed Thermography (IRT)

Constrained IRT

EBSD analysis

Displacement and strain mapping

Crystal plasticity

\begin{abstract}
A B S T R A C T
With the intention of achieving an experimental grain scale energy balance at finite strain and at the grain scale, a mechanical test on a coarse-grained aluminium is presented in this paper using two complementary imaging techniques based on visible and infrared light. Specific image processing methods referred to as Constrained Digital Image Correlation (Constrained DIC) and Constrained InfraRed Thermography (Constrained IRT) are applied to investigate the thermomechanical behavior at the microstructural scale. Constrained DIC is used to obtain displacement and strain fields during the test, while Constrained IRT provides an estimate of temperature and heat source fields induced by the mechanical loading. The proposed "constrained" methods allow to enforce an adjustable level of constraints on a measured field (displacement or temperature) without referring to a specific finite-element description. In that manner, it is possible to decouple the measurement model and the interpretation model while keeping regularizing constraints (such as continuity of the fields). In this paper, we mainly focus on the kinematic analysis of the experimental test. Electron Backscatter Diffraction (EBSD) is also used in this case to experimentally characterize the microstucture of a $3 \mathrm{~mm}$ thick specimen with centimetric grain size.
\end{abstract}

\section{Introduction}

Polycrystalline metals usually possess a microstructure composed of an aggregation of crystalline grains with varying size, morphology and orientation. During a macroscopic tensile loading, the diversity of grain orientations and the intrinsic anisotropy of crystal plasticity leads to strong heterogeneities in the material plastic response, and consequently to an inhomogeneous thermal distribution due to thermomechanical effects.

Recently, heterogeneous phenomena on mechanical and thermal fields have been studied in metallic materials at the granular scale [16]. All these works have shown the variety of micromechanical modelling issues that can be addressed using classical DIC (Digital Image Correlation) \& IRT (InfraRed Thermography) method. Hereafter, a "Constrained" surface DIC or IRT method is proposed to enrich the kinematic or thermal transformation of neighbouring elements (or grains) by imposing continuity (or discontinuity) conditions on the displacement (or the displacement gradient component) or on the temperature (or the temperature gradient).

Performing strain field and heat source measurements ultimately allows to access to the evolution of the mechanical and calorimetric en- ergies involved in the transformation. This assessment contributes to a better knowledge of the local thermomechanical signature of the material deformation mechanisms.

As mentioned, two data processing methods (Constrained DIC $[7,8]$ and Constrained IRT [9]) are required to perform kinematic and thermal measurements that are both needed to conduct a local energy balance within each grain during a mechanically-loaded test. In the light of this general objective, we mainly focus in this paper on the kinematic aspect of the aforementioned general methodology.

First, the principle of Constrained DIC method will be introduced. Then, the numerical validation of Constrained DIC method will be performed on numerical example associated to cracked polycrystalline aggregates. Afterwards, this novel method will be applied to real experimental images.

In fact, surface displacement field measurements of materials subjected to various loadings (e.g. mechanical loading or thermal loading) are an important task for experimentalists addressing challenges in the field of solid mechanics.

In recent years, an increasing number of spectacular developments in optical full-field measurement techniques has been witnessed [10], including both interferometric techniques and non-interferometric techniques. However, the interferometric techniques involve delicate proce- 
dures which are not always easily transferable to conventional testing laboratories. Consequently, the Digital Image Correlation (DIC) method widely considered as a representative non-interferometric optical technique, has been largely accepted and commonly used as a powerful and flexible tool for surface displacement and strain measurement in the experimental solid mechanics field $[2,4,6,11-14]$.

These measurements are particularly valuable in the sense that they allow the interpretation of complex tests at different scales and that they are naturally adapted to scale transitions. For these reasons, they have been largely used to characterise the deformation mechanisms or to propose and validate micromechanical models or scale transition laws.

From a microstructural viewpoint, polycrystalline materials are a discrete structure that are composed of jointed grains with varying sizes and orientations. The characterisation and measurement of grain structures is of great interest to Materials Scientists because they are directly related to the physical properties of matter $[15,16]$.

Our objective here is the understanding of the relationship between the microstructural parameters and the mechanical behaviour of the heterogeneous materials at the macroscopic scale, in particular at the granular length scale [17-19].

Using the classical local approaches, the material microstructure is not accounted for in the kinematic computation:

- Firstly, the introduced subsets (for DIC) are independently defined from the microstructure

- Secondly, as the transformation of neighbouring subsets are separately processed, so subsets may overlap.

This is an inherent disadvantage of these local methods when dealing with heterogeneous structure problems.

Nevertheless, classical local DIC methods have been widely used to highlight the heterogeneity in kinematic fields $[2,6,12,20]$, in a large range of situations dealing for instance, with the fracture mechanics (intergranular or intragranular) problems.

\section{Principle of constrained DIC method}

Global DIC methods were proposed to determine the displacement and strain fields on the whole image. These methods propose to parametrize the kinematic fields using a limited set of degrees of freedom which tends to regularize the DIC problem. These methods were firstly introduced to impose the continuity of measured displacement on a finite-element mesh $[21,22]$ or using B-splines [23,24]. Gobal DIC methods were afterwards extended to allow some discontinuities in the displacement fields to account for crack development $[25,26]$.

The Constrained DIC method proposed here corresponds to an alternative to global DIC methods. It relies on a mesh that respects the material microstructure and it introduces shape functions that are expressed in the real space and not on the associated reference element (as in classical finite elements). The shape functions can be any kind (we generally use linear, bi-linear, quadratic, bi-quadratic polynomial functions), and the shape function choice is independent of the shape of the element. The most significant difference with global (finite-element based) DIC methods relies in the fact that the level of restriction between two adjacent elements can be modified by choosing the number (and the location) of points where to enforce the continuity conditions on the element boundary. It is also interesting to note that the proposed method allows to handle in the same framework classical local DIC methods (which corresponds to a regular rectangular mesh with no continuity condition between each elements) to global finite-element based methods on regular meshes (by imposing continuity conditions on the ends of each element boundary).

As classical DIC approaches (whether local or global), the proposed method also relies on the Brightness Conservation equation [27] motivating the use of a pattern recognition algorithm for the detection of changes in the grey level distribution of targeted surface during loading. Indeed, the main steps of Constrained DIC method are the following:

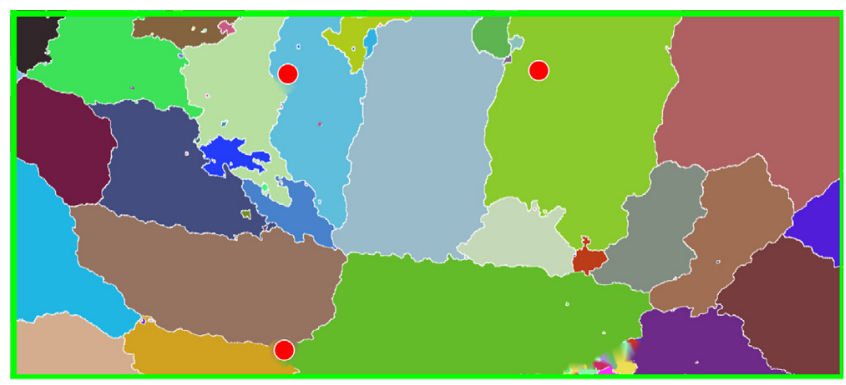

(a) Microstructure of a polycrystalline metallic material analysed by EBSD

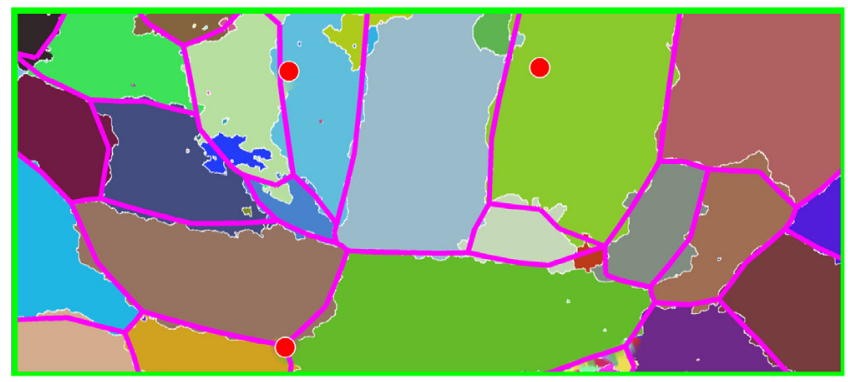

(b) Grain boundaries extraction

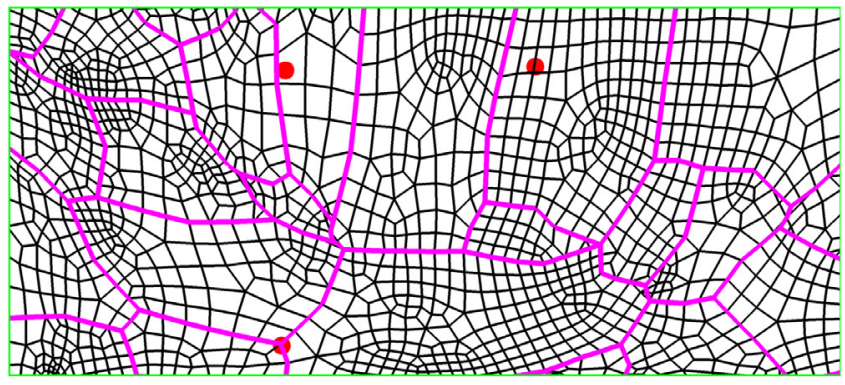

(c) Unstructured mesh for this polycrystalline material

Fig. 1. Spatial description of the geometry of a polycrystalline aggregation. The grain boundaries are in magenta and the element contours are in black. And the three red dots are for spatial matching procedure [6]. (For interpretation of the references to color in this figure legend, the reader is referred to the web version of this article.)

- Spatial discretization of the geometry

Through an EBSD analysis, a two-dimensional array of data associated with the microstructure is provided by microscopic devise $[28,29]$. Afterwards, this microstructural map (Fig. 1a) can be used to perform a spatial discretization (Finite Element type) in order to respect as much as possible the real microstructure. The obtained mesh is used for subsequent processing of the kinematic response. In order to optimize the meshing procedure, the real grain boundaries (white contours in Fig. 1a) are simplified and polygonized so as to keep the large grains and regroup the smallest ones, as shown in Fig. 1b in magenta. By construction, the level of microstructural simplification has to be adjusted depending on the spatial resolution associated with the kinematic and/or thermal measurement. The introduced uncertainty during the grain boundary extraction operation is not quantified, which is supposed to be negligible in this paper. Afterwards, an unstructured mesh is carefully applied on the "simplified" geometry (representing the microstructure) within each grain in order to keep the representation of physical grain boundaries, as shown in Fig. 1c. Inside each grain, the smallest mesh unit is called an "element", which is equivalent of the correlation subset for classical DIC methods. The element contours are accurately determined. 
The computational mesh underlying the microstructure is defined in the initial configuration. The kinematic variables associated with each element describing the physical transformation of the material will be introduced in the next section.

- Description of the physics

The specimen might be subjected to different loadings (traction, compression, shear or rotation). Depending on the mechanical situation under consideration, the displacement field can be continuous (continuous medium) or discontinuous (granular medium or fracture). The method developed here proposes to enrich the DIC formulation in order to introduce constraints in the DIC algorithms compatible with the continuity or discontinuity of displacement field.

In order to describe the kinematic physics, a polynomial shape function is assigned to each element $\mathbf{e}$ of the mesh to represent the local displacement variations (Eq. (1)).

$$
\begin{aligned}
& \mathbf{u}_{\mathbf{x}}^{\mathbf{e}}\left(X_{C C D}, Y_{C C D}, p_{X}^{\mathbf{e}}\right)=\sum_{k=0}^{d k_{X}} \sum_{l=0}^{d l_{X}} a_{k l}^{\mathbf{e}} X_{C C D}^{k} Y_{C C D}^{l} \\
& \mathbf{u}_{\mathbf{y}}^{\mathbf{e}}\left(X_{C C D}, Y_{C C D}, p_{Y}^{\mathbf{e}}\right)=\sum_{k=0}^{d k_{Y}} \sum_{l=0}^{d l_{Y}} b_{k l}^{\mathbf{e}} X_{C C D}^{k} Y_{C C D}^{l}
\end{aligned}
$$

where, $\mathbf{u}_{\mathbf{x}}^{\mathbf{e}}$ and $\mathbf{u}_{\mathbf{y}}^{\mathbf{e}}$ are the components of local displacement field $\left(\mathbf{u}^{\mathbf{e}}\right)$ for element $\mathbf{e}$ in the directions $\vec{X}$ and $\vec{Y}$, and $\left(X_{C C D}, Y_{C C D}\right)$ represents the Lagrangian coordinates in pixels. Vector $\mathbf{p}^{\mathbf{e}}=\left(p_{X}^{\mathbf{e}}, p_{Y}^{\mathbf{e}}\right)=\left(a_{k l}^{\mathbf{e}}, b_{k l}^{\mathbf{e}}\right)$ gathers the kinematic shape function parameters. The order and the type of the kinematic shape function can be chosen according to requirements. The displacement fields in each element are thus described in the real space, and not through a "reference element", which is the case for classical Finite Element (FE) descriptions $[25,30]$.

- Introduction of the restriction

In the previous section, kinematic transformations for each individual element have been introduced. We will now detail the possible relationships between kinematic transformation of neighbouring elements.

Three situations can be encountered:

- The transformations between neighbouring elements are completely independent.

- At least one component of the displacement vector is continuous through the common boundary.

- A displacement jump is allowed on the common boundary. The first situation corresponds to classical local DIC methods. When imposing continuity conditions on both displacement components, the second situation is analogous to global DIC methods [31]. Here, the continuity condition can be enforced on the whole boundary (exact restriction) or in a limited number of nodes (partial restriction). In the same spirit, continuity conditions can also be introduced at boundary on the displacement derivatives. The third one corresponds to unilateral condition (crack opening). This kind of restrictions will not discussed in this paper.

After the displacement shape function has been chosen for each element, restrictions can be introduced between the kinematic fields associated with each pair of neighbouring elements. Fig. 2 schematically illustrates the situation for a given pair of adjacent elements (element $i$ and element $j$ ) of the kinematic mesh. As proposed, the degrees of the polynomials for kinematic description in element $i$ and element $j$ are not necessarily identical.

The boundary $l_{u}^{i j}$ between elements $i$ and $j$ is modeled as a linear relationship between $X$ and $Y$, whose coefficients depend only on the mesh geometry. For a relatively "horizontal" boundary, as shown in Fig. 2, the boundary $l_{u}^{i j}$ is expressed as

$Y_{C C D}=\alpha_{u} X_{C C D}+\beta_{u}$

Naturally, the case of a "vertical" boundary is deduced by inverting the role of $X$ and $Y$, expressed as $X_{C C D}=\alpha_{u}^{\prime} Y_{C C D}+\beta_{u}^{\prime}$.

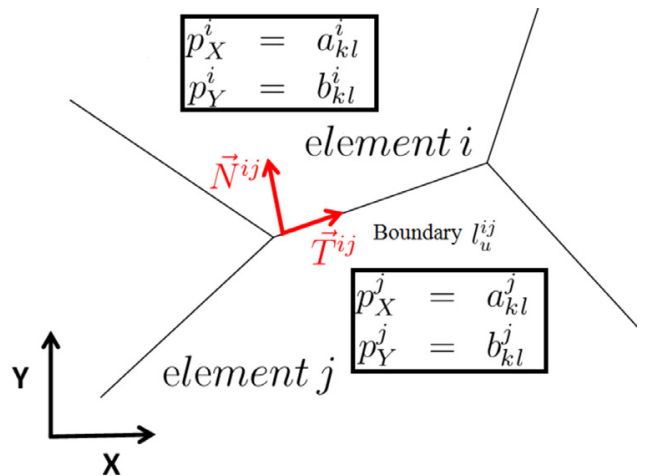

Fig. 2. Description of the boundary $l_{u}^{i j}$ between two adjacent elements $i$ and $j$ and definition of the local Normal-Tangential coordinate system $\left(\vec{N}^{i j}, \vec{T}^{i j}\right)$ of the boundary $l_{u}^{i j}$.

By construction, the coefficients $\left\{\alpha_{u}, \beta_{u}\right\}$ or $\left\{\alpha_{u}^{\prime}, \beta_{u}^{\prime}\right\}$ of the boundary expression are defined only by the geometrical mesh. The components of the normal and tangential vectors of the boundary, $\vec{N}^{i j}$ and $\vec{T}^{i j}$, are expressed from $\alpha_{u}$ or $\alpha_{u}^{\prime}$ as follows:

- for the relatively "horizontal" boundary:

$$
\vec{N}^{i j}=\left(\begin{array}{c}
-\alpha_{u} \\
1
\end{array}\right) \text { and } \vec{T}^{i j}=\left(\begin{array}{c}
1 \\
\alpha_{u}
\end{array}\right)
$$

- for the relatively "vertical" boundary:

$$
\vec{N}^{i j}=\left(\begin{array}{c}
1 \\
-\alpha_{u}^{\prime}
\end{array}\right) \text { and } \vec{T}^{i j}=\left(\begin{array}{c}
\alpha_{u}^{\prime} \\
1
\end{array}\right)
$$

The restriction conditions are introduced along the element boundary $l_{u}^{i j}$ using the local Normal-Tangential coordinate system $\left(\vec{N}^{i j}, \vec{T}^{i j}\right)$ of the boundary. As mentioned above, different kind of restrictions can be imposed:

- continuity restriction: equality of the variable (or its derivative) on both sides of the boundary

- jump restriction: inequality of the variable (or its derivative) on both sides of the boundary

In this paper, we only focused on describing continuity restrictions. They correspond to the introduction of linear equations between the parameters describing kinematic fields of two adjacent elements. The continuity of the displacement field $\left(\mathbf{u}^{\mathbf{e}}\right)$ is imposed in the local Normal-Tangential coordinate system of the boundary, in order to impose either a normal or a tangential displacement continuity (or both simultaneously).

Furthermore, restriction conditions can also be imposed on the displacement gradient on the element boundaries.

Finally, taking into account these different restrictions leads to impose the corresponding linear equations between the two adjacent elements $i$ and $j$, that can be expressed as a linear system:

$\left[\mathbf{A}_{\mathbf{U}}^{\mathbf{i j}}\right]\left\{\mathbf{P}_{\mathbf{U}}^{\mathbf{i j}}\right\}=\{\mathbf{0}\}$

where $\left[\mathbf{A}_{\mathbf{U}}^{\mathrm{ij}}\right]$ is the kinematic elementary restriction matrix between element $i$ and $j$, and $\left\{\mathbf{P}_{\mathbf{U}}^{\mathbf{i j}}\right\}$ is the elementary vector containing all the unknown kinematic parameters $\left(\mathbf{p}^{\mathbf{i}}, \mathbf{p}^{\mathbf{j}}\right)=\left(a_{k l}^{i}, b_{k l}^{i}, a_{k l}^{j}, b_{k l}^{j}\right)$ for these two adjacent elements. The linear relations between $\mathbf{p}^{\mathbf{i}}$ and $\mathbf{p}^{\mathbf{j}}$ (Eq. (5)) allows to decrease the number of independent parameters to be determined by correlation for element $i$ and $j$. The introduction of this linear relations reduces the number of Degrees Of Freedom (DOFs) required to describe the kinematic field.

By iterating this operation for all boundaries on which continuity restrictions are applied, a global kinematic restriction matrix $\mathbf{A}_{\mathbf{U}}$ is built for the mesh, as well as a global vector $\mathbf{P}_{\mathbf{U}}$ containing all the kinematic parameters.

$\left[\mathbf{A}_{\mathbf{U}}\right]\left\{\mathbf{P}_{\mathbf{U}}\right\}=\{\mathbf{0}\}$ 
The line number of $\mathbf{A}_{\mathbf{U}}$ corresponds the introduced restriction number. The column number of this matrix is related to the number of parameter for the kinematic description. The rank of the restriction matrix is necessarily smaller than the number of parameter, so this matrix can be triangularized to take out the "independent" parameters for the kinematic description.

It is important to recall that, working in the Lagrangian framework, all restriction matrices are determined in the initial configuration and are calculated only once throughout the data processing.

- Resolution of the enriched kinematic problem

As mentioned in the beginning of this section, this Constrained DIC problem is solved under the principle of Brightness Conservation [27].

A correlation criterion is chosen to quantify the luminance mismatch difference between the initial and final images. Different correlation criterion can be introduced as shown in the literature [14,32]. Here, we used the SSD criterion (Sum of Squared Differences) to assess the similarity between the reference image $I_{0}$ and the deformed image $I_{1}$ :

$$
\begin{aligned}
& \mathfrak{夭}_{S S D}=\sum_{\mathbf{e}} \iint_{S_{\mathbf{e}}}\left[I_{0}\left(X_{C C D}, Y_{C C D}\right)\right. \\
& -I_{1}\left(X_{C C D}+\mathbf{u}_{\mathbf{x}}^{\mathbf{e}}\left(X_{C C D}, Y_{C C D}, p_{X}^{e}\right)\right. \\
& \left.\left.Y_{C C D}+\mathbf{u}_{\mathbf{y}}^{\mathbf{e}}\left(X_{C C D}, Y_{C C D}, p_{Y}^{e}\right)\right)\right]^{2} \mathrm{~d} X_{C C D} \mathrm{~d} Y_{C C D}
\end{aligned}
$$

where, $S_{\mathrm{e}}$ represents the polygon associated with the element "e", $\mathbf{u}_{\mathbf{x}}^{\mathrm{e}}$ and $\mathbf{u}_{\mathbf{y}}^{\mathbf{e}}$ represent the shape functions which depend on parameters $p_{X}^{e}$ and $p_{Y}^{e}$.

The numerical resolution of this kinematic problem consists in minimizing the functional $\left(\mathfrak{c}_{S S D}\right)$ under the above mentioned linear constraint.

$$
\begin{gathered}
\mathbf{P}_{\mathbf{U}}^{\text {optimal }}=\underset{p_{X}, p_{Y}}{\operatorname{argmin}}\left\{\mathfrak{\complement}_{S S D}\right\} \\
\text { with }\left[\mathbf{A}_{\mathbf{U}}\right]\left\{\mathbf{P}_{\mathbf{U}}\right\}=\{\mathbf{0}\}
\end{gathered}
$$

By construction, the global vector $\mathbf{P}_{\mathbf{U}}$ has been previously obtained by assembling all the elementary vector of each element. Once this global vector $\mathbf{P}_{\mathbf{U}}^{\text {optimal }}$ is determined, all the elementary vector $\left(\mathbf{P}_{\mathbf{U}}^{\mathbf{e}}\right)$ are determined, allowing to describe the local displacement fields $\overrightarrow{\mathbf{u}}^{\mathrm{e}}=\left(\begin{array}{c}\mathbf{u}_{\mathbf{x}}^{\mathrm{e}} \\ \mathbf{u}_{\mathbf{y}}^{\mathrm{e}}\end{array}\right)$ for each element.

The components of the local finite transformation gradient tensor $\boldsymbol{F}^{\mathbf{e}}$ for each element $\mathbf{e}$ is then expressed analytically:

$$
\left\{\begin{array}{l}
\boldsymbol{F}_{x X}^{e}=1+\frac{\partial \mathbf{u}_{\mathbf{x}}^{\mathbf{e}}}{\partial X} \\
\boldsymbol{F}_{x Y}^{e}=\frac{\partial \mathbf{u}_{\mathbf{x}}^{\mathbf{e}}}{\partial Y} \\
\boldsymbol{F}_{y X}^{e}=\frac{\partial \mathbf{u}_{\mathbf{y}}^{\mathbf{e}}}{\partial X} \\
\boldsymbol{F}_{y Y}^{e}=1+\frac{\partial \mathbf{u}_{\mathbf{y}}^{\mathbf{e}}}{\partial Y}
\end{array}\right.
$$

Strain fields are deduced in Lagrangian, Eulerian or small deformation framework.

Afterwards, these local quantities can be analytically integrated to obtain the average values per element. Similarly, by defining element sets belonging to one grain, these quantities can be averaged for each grain.

Using the method that has been presented above, the kinematic data can be analysed with different restrictions. As the restrictions are imposed between two elements, we can naturally process intergranular or intragranular crack data. In addition, instead of the restriction imposed upon all the boundary points (exact restriction), it is possible to impose

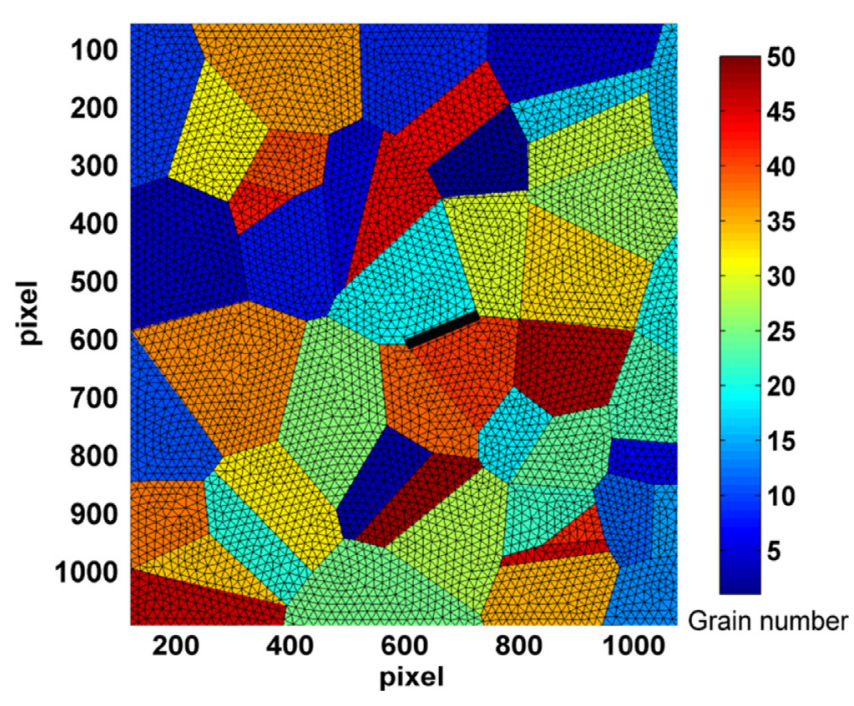

Fig. 3. Microstructure of a polycrystalline aggregation used for FE simulation.

it on a limited number of nodes on the element boundary (partial restriction), resulting in less constraint equations and thus a more compliant kinematic description.

After having introduced the principle and some advantages of Constrained DIC method, the performance of this kinematic data processing method will be demonstrated through numerical examples on polycrystalline aggregates in the next section. Concerning the thermal data processing, the Constrained IRT method has been previously presented in [9].

\section{Numerical validation on a cracked specimen}

The procedure is numerically validated on heterogeneous fields obtained on computer-generated speckle images associated with completely known kinematic fields: here, the focus will be an intergranular crack opening in a polycrystalline microstructure.

\subsection{Synthetic image generation}

Crystal plasticity is usually used in materials science as the constitutive model to describe the response of crystal grains. The objective of crystal plasticity is to introduce slip elementary features into the description of plasticity [33]. At the same time, one major interest of micromechanics of heterogeneous polycrystalline materials is to access local mechanical fields in a given microstructure associated to surface strain fields that can be measured [17-19], in order to contribute to a better understanding of the microstructure dependence of yield behaviour during the mechanical loading at granular scales, and to assess local stress fields in view of developing physically-based damage models.

For this numerical study, the kinematic field was obtained by direct crystal plasticity Finite Element (FE) analysis for the crystal plasticity law proposed by Cailletaud and Meric [34] and for a given set of boundary conditions and grain orientations. An aggregate of 50 grains was generated using a classical Vorono tessellation [35-38], which provides convex polyhedric grains as shown in Fig. 3. This microstructure is representative of coarse-grained aluminum having relatively equiaxed grains of convex shape. The size distribution of grains has been randomly chosen but could be adjusted more precisely if needed. In order to focus on intergranular cracking, an initial intergranular crack is introduced in the middle of the specimen (colored in black in the middle of Fig. 3).

The chosen material behaviour obeys the Méric-Cailletaud model, described in [34] for FCC slip systems. The boundary conditions have 


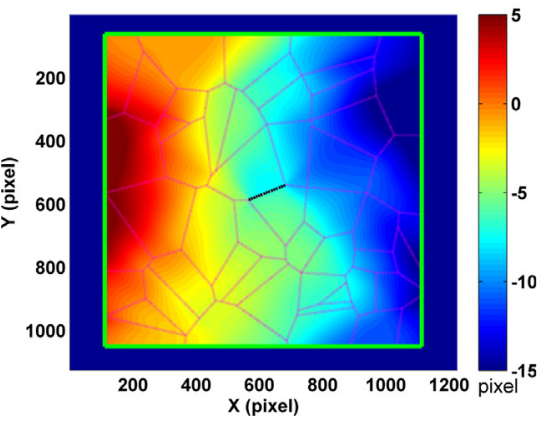

(a) Transverse compenent of the theoretical displacement field $\mathbf{u}_{\mathbf{x}}^{\text {Aster }}$

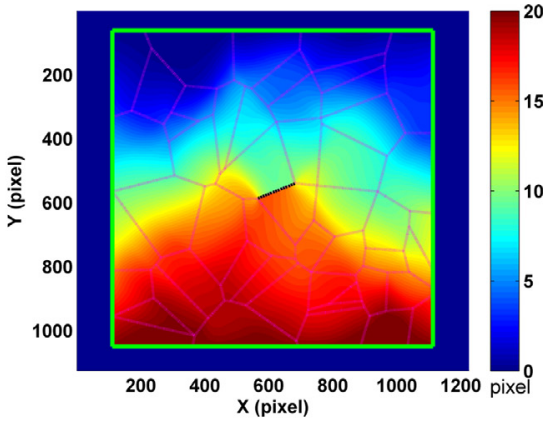

(b) Axial compenent of the theoretical displacement field $\mathbf{u}_{\mathbf{y}}^{\text {Aster }}$

Fig. 4. Displacement fields obtained by FE simulation.

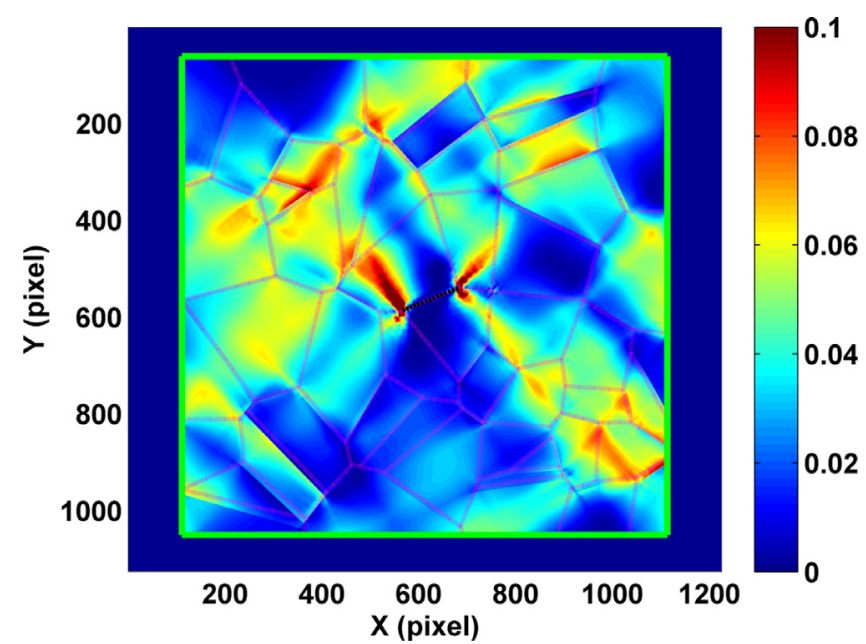

Fig. 5. Theoretical equivalent Von Mises strain field for the cracked polycrystal aggregates $\varepsilon_{\mathrm{eq}}^{\text {Aster }}$.

been prescribed using uniform displacement at the upper and lower edges of the mesh, and the grain orientations of the microstructure are randomly chosen to obtain an isotropic texture (and therefore an isotropic tensile response). Finally, the numerical simulation was performed using the FE package Code_Aster with a mesh of 11,300 quadratic triangular elements (Fig. 3) in a bi-dimensional framework under a plane strain assumption [39].

The aim of this FE computation is to provide realistic local kinematic fields associated with equilibrated stress fields and corresponding to realistic strain and stress heterogeneities.

The intergranular crack generates a discontinuity, in the displacement field on both components, as shown in Fig. 4.

A $2 \%$ macroscopic strain is imposed for this numerical study. The strain field is highly localized around the crack tip, with 2D plastic equivalent strain ${ }^{1}$ exceeding $12 \%$ (Fig. 5).

The simulated displacement field was introduced in a virtual image generation procedure, as described in [12], to mimic the acquisition of a serie of speckle images. The initial image with the superimposed initial microstructure is presented in Fig. 6. It was chosen not to introduce any artificial image distortion in the synthetic images procedure, in order to focus solely on the DIC algorithm biases.

\footnotetext{
${ }^{1}$ The equivalent Von Mises strain is defined here by: $\varepsilon_{\mathrm{eq}}=$ $\sqrt[2]{\frac{2}{3}\left(\varepsilon_{x x}^{2}+2 \times \varepsilon_{x y}^{2}+\varepsilon_{y y}^{2}\right)}$
}

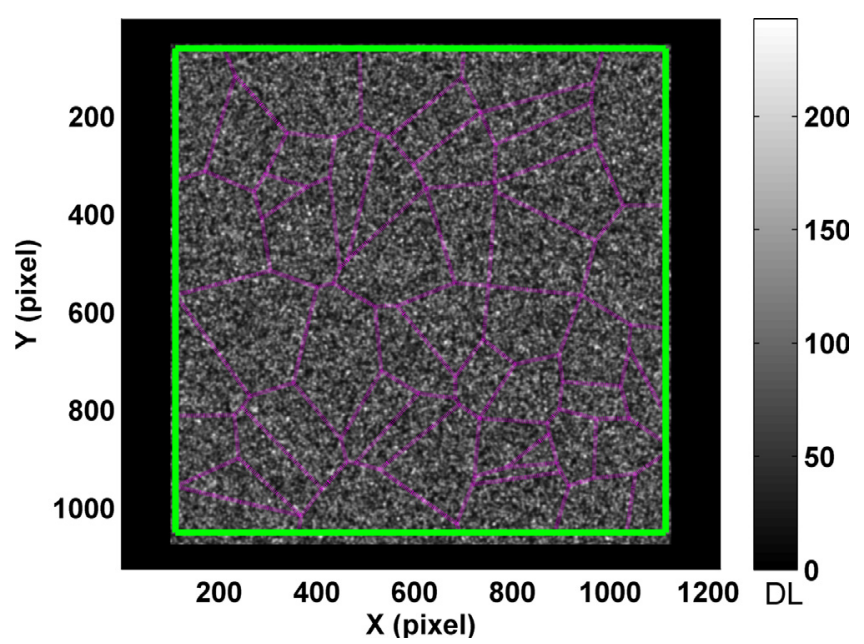

Fig. 6. Reference synthetic image.

To better illustrate the intergranular crack development in the synthetic image, a magnified view of the cracking zone is presented respectively for the reference image (Fig. 7a) and the deformed image (Fig. 7b).

During the crack opening, the optical flow is not conserved between the crack lips. To account for this phenomenon, a mask is superimposed in the deformed image. This mask consists in a black line with a varying width equals to the computed local crack opening displacement (see Fig. $7 \mathrm{~b}$ in the middle).

The robustness of DIC methods with respect to experimental noise is always a key issue $[32,40]$. Here, by decreasing the number of independent degrees of freedom used to describe the displacement fields, the proposed method diminishes the sensitivity to measurement noise, but it adds spatial correlations in the displacement fields due to the linear constraints between the degrees of freedom. To study the noise propagation in the method, we propose to introduce noise in these virtual images in order to account for the different error sources in the acquisition chain, as proposed in [32]. Such study will be the subject of a forthcoming publication.

\subsection{Numerical results}

This section presents results obtained using various combinations of processing parameters. Working with images with perfectly known deformation allows to quantify the processing error by comparing the kinematic fields obtained by Constrained DIC to that derived from FE simulation. 


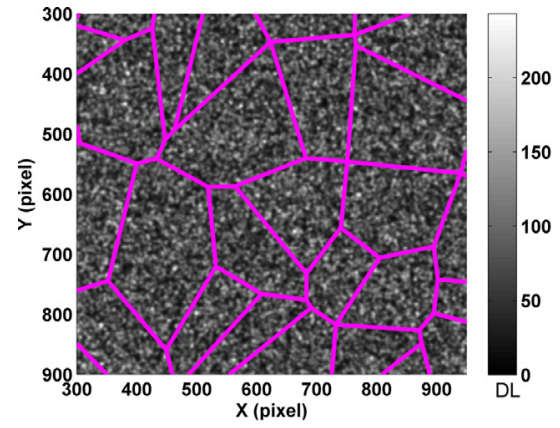

(a) Zoom of reference image

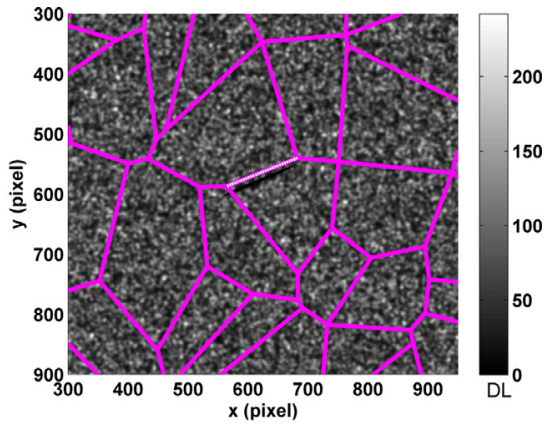

(b) Zoom of deformed image

Fig. 7. Zoom of the crack tip in the reference image and the deformed image.

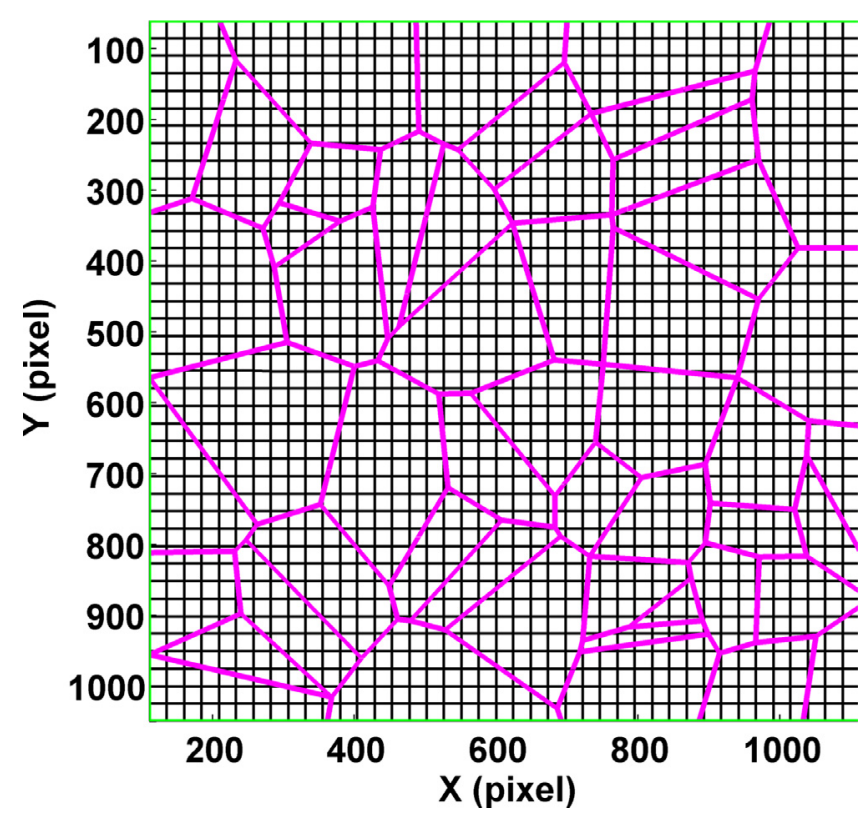

Fig. 8. Regular mesh for DIC processing.

\subsubsection{Kinematic validation}

In this part, three processing results are presented. The three combinations of parameters associated with these three processing cases are precised below:

- processing parameters for study-I:

- Regular mesh (with 1640 elements), as shown in Fig. 8.

- No continuity restriction between any element.

- Bi-linear shape function (with respect to both variables, $X$ and $Y$ ) for kinematic description of each element (8 parameters per element), as introduced in Eq. (10).

$\mathbf{u}_{\mathbf{x}}\left(X, Y, p_{X}\right)=a_{00}+a_{10} X+a_{01} Y+a_{11} X Y$

$\mathbf{u}_{\mathbf{y}}\left(X, Y, p_{Y}\right)=b_{00}+b_{10} X+b_{01} Y+b_{11} X Y$

- The associated number of DOFs introduced to describe the kinematic field is then $8 \times 1640=13120$

- processing parameters for study-II:

- Microstructural mesh (with 1513 elements), as shown in Fig. 9. This mesh is consistent with the material microstructure.

- No continuity restriction between any element.

- Bi-linear shape function as introduced in Eq. (10).

- DOFs $=8 \times 1513=12104$

- processing parameters for study-III:

- Microstructural mesh, as shown in Fig. 9.

- Exact continuity restriction applied to all adjacent elements within each grain. These restrictions impose intragranular conti-

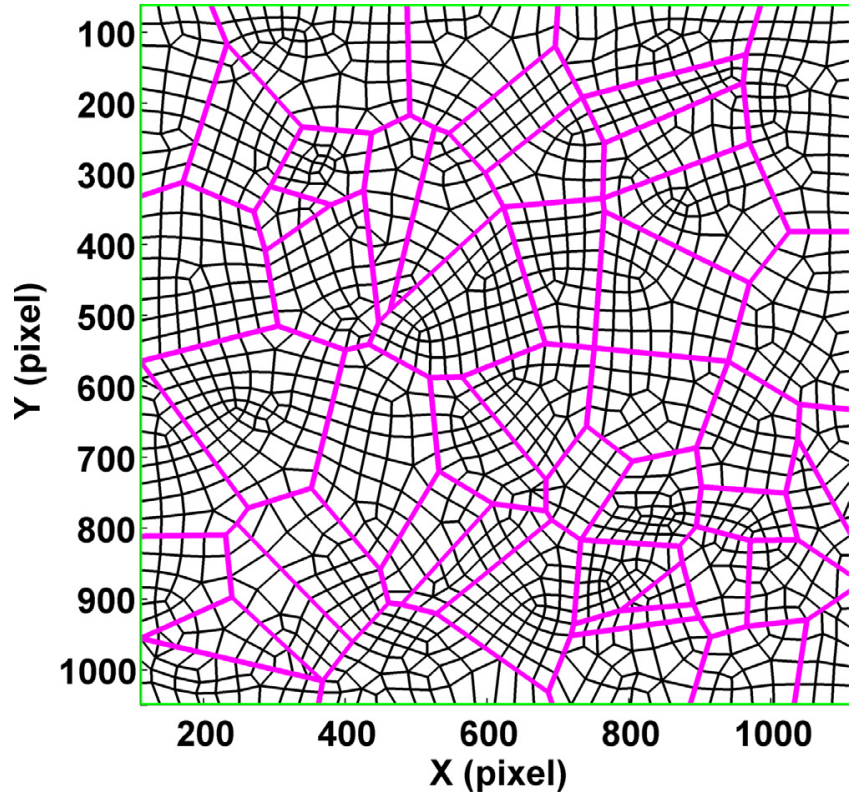

Fig. 9. Microstructural mesh for DIC processing.

nuity, but they allow the development of intergranular discontinuity (cracking).

- Bi-linear shape function as introduced in Eq. (10).

- DOFs $=1372$

The study-I is equivalent to a classical local DIC. The study-II is a equivalent to a classical local DIC using a non regular mesh consistent with the microstructure. The study-III is a Constraint DIC analysis with imposed intragranular continuity restriction.

Naturally it is important to quantify the data processing error with the proposed method. The error is defined as the difference between the calculated results and their imposed "theoretical" values. In order to facilitate the interpretation of error maps, we will only focus on equivalent Von Mises strain field $\left(\varepsilon_{\mathrm{eq}}\right)$, averaged on each element.

The reference field for both regular and microstructural meshes are obtained by integrating the FE-computed equivalent strain on each mesh. They are represented in Fig. 10.

Figs. 11-13 show the error distributions for the aforementioned three studies. The Mean Error (ME) and its Standard Deviation (SD) are reported in Eqs. (11)-(13).

$$
\begin{aligned}
& \left\{\begin{array}{l}
\operatorname{ME}\left(\varepsilon_{\text {eq }}^{\text {Study-I }}-\varepsilon_{\text {eq }}^{\text {Aster }}\right)=-0.59 \times 10^{-4} \\
\operatorname{SD}\left(\varepsilon_{\text {eq }}^{\text {Study-I }}-\varepsilon_{\text {eq }}^{\text {Aster }}\right)=6 \times 10^{-3}
\end{array}\right. \\
& \left\{\begin{array}{l}
\operatorname{ME}\left(\varepsilon_{\text {eq }}^{\text {Study-II }}-\varepsilon_{\text {eq }}^{\text {Aster }}\right)=-1.581 \times 10^{-4} \\
\operatorname{SD}\left(\varepsilon_{\text {eq }}^{\text {Study-II }}-\varepsilon_{\text {eq }}^{\text {Aster }}\right)=6 \times 10^{-3}
\end{array}\right.
\end{aligned}
$$




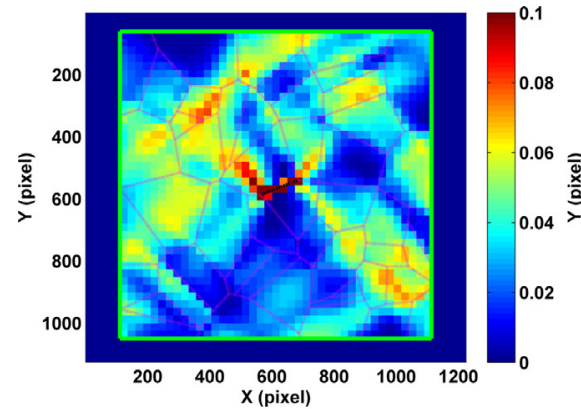

(a) Equivalent Von Mises strain field averaged according regular mesh

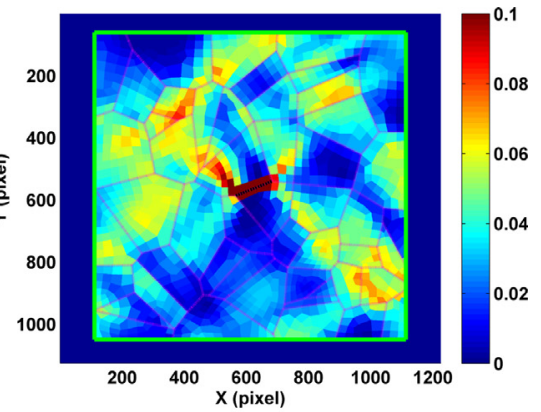

(b) Equivalent Von Mises strain field averaged according microstructural mesh

Fig. 10. Averaged equivalent strain fields obtained by FE simulation.

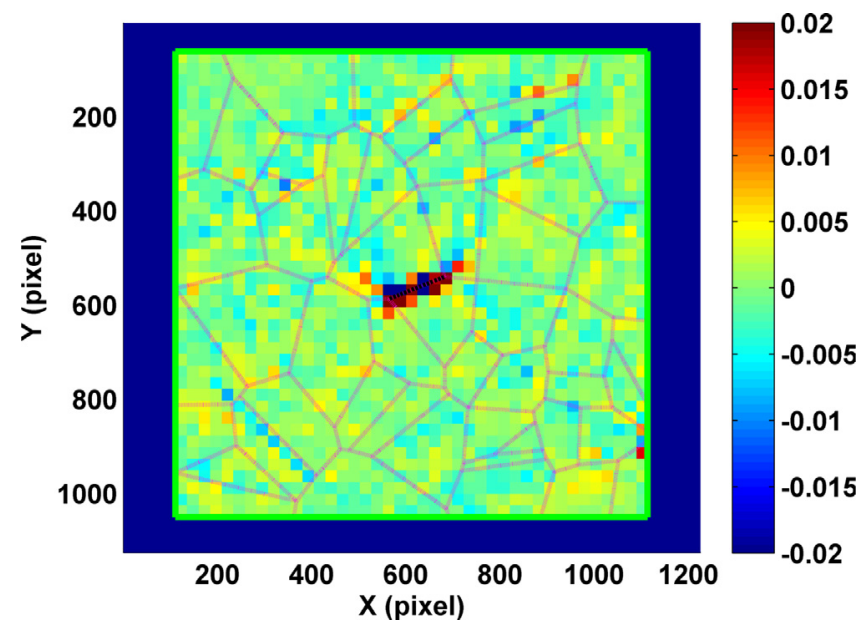

Fig. 11. Element-averaged error map $\left(\varepsilon_{\text {eq }}^{\text {Study-I }}-\varepsilon_{\text {eq }}^{\text {Aster }}\right)$ for Study-I.

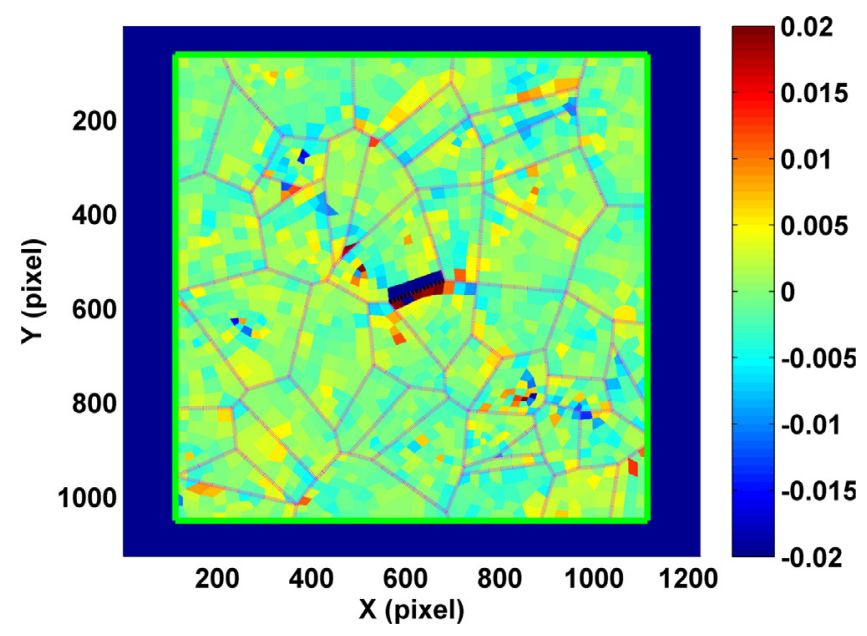

Fig. 12. Element-averaged error map $\left(\varepsilon_{\mathrm{eq}}^{\text {Study-II }}-\varepsilon_{\mathrm{eq}}^{\text {Aster }}\right)$ for Study-II.

$\left\{\begin{array}{l}\operatorname{ME}\left(\varepsilon_{\text {eq }}^{\text {Study-III }}-\varepsilon_{\text {eq }}^{\text {Aster }}\right)=3.327 \times 10^{-4} \\ \operatorname{SD}\left(\varepsilon_{\text {eq }}^{\text {Study-III }}-\varepsilon_{\text {eq }}^{\text {Aster }}\right)=8 \times 10^{-3}\end{array}\right.$

The error distributions are very different from one analysis to another, but they correspond to very similar global levels in Mean Error (ME) or Standard Deviation (SD).

It is important to point out that, for an equivalent level of performance, Study-III uses ten times less DOFs than the two others. Consequently, Study-III will be much more robust against the noise.

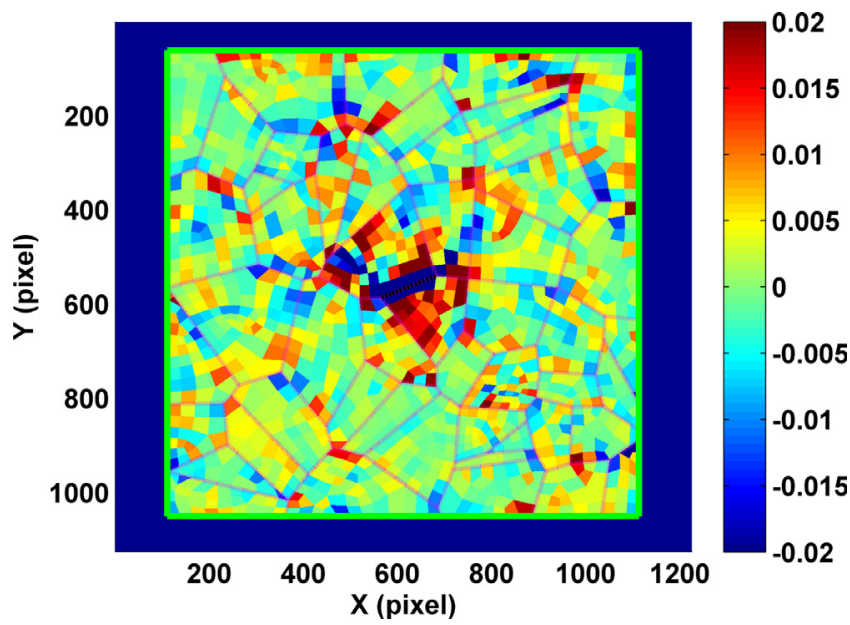

Fig. 13. Element-averaged error $\operatorname{map}\left(\varepsilon_{\mathrm{eq}}^{\text {Study-III }}-\varepsilon_{\mathrm{eq}}^{\text {Aster }}\right)$ for Study-III.

The strain errors are highly localized around the crack tip for the three studies. The first reason is due to the non conservation optic flow in this region. The second one is the presence of strong strain gradients at the rear of crack tip. Everywhere else, the error level is smaller and is randomly distributed.

The Study-III gives the highest error levels because of the reduced number of DOFs, that generates a coarser kinematic description.

\subsubsection{Crack description}

A local analysis will now be conducted focusing on the crack lips. The displacement jump profiles computed for the different methods will be used to quantify their performances in capturing discontinuities.

Indeed, the displacement jump is defined in the local NormalTangential coordinate system of the crack as the difference between the measured displacement in the lower side of the crack and in the upper side.

Fig. 14represents the evolution of the normal displacement jump for the different processing along the crack. In this figure, the FE-simulated displacement jump profile (Aster) gives the reference in red.

The results obtained for Study-I (green one in Fig. 14) are very different from the reference ones. Indeed, in this case, the mesh is regular and is not consistent with the microstructure, so the crack crosses the elements used for DIC processing. Furthermore, the shape functions are continuous within each element, but they are discontinuous from on element to another (i.e. on each element boundary). Consequently, the discontinuity remains very challenging to capture in this case.

However, the Study-II (local DIC with microstructural mesh) provides a slightly closer displacement jump profile (in cyan) to the reference one. In fact, the crack physically contains 5 elements. And the important displacement jumps are found at each element changing, since no continuity restriction is imposed along the crack between the ele- 


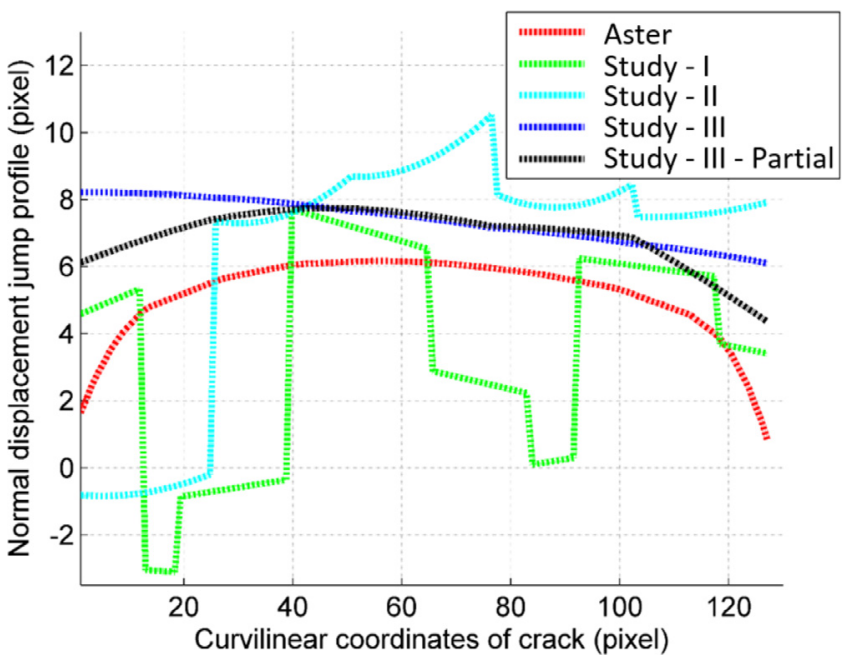

Fig. 14. Normal displacement jump profile.

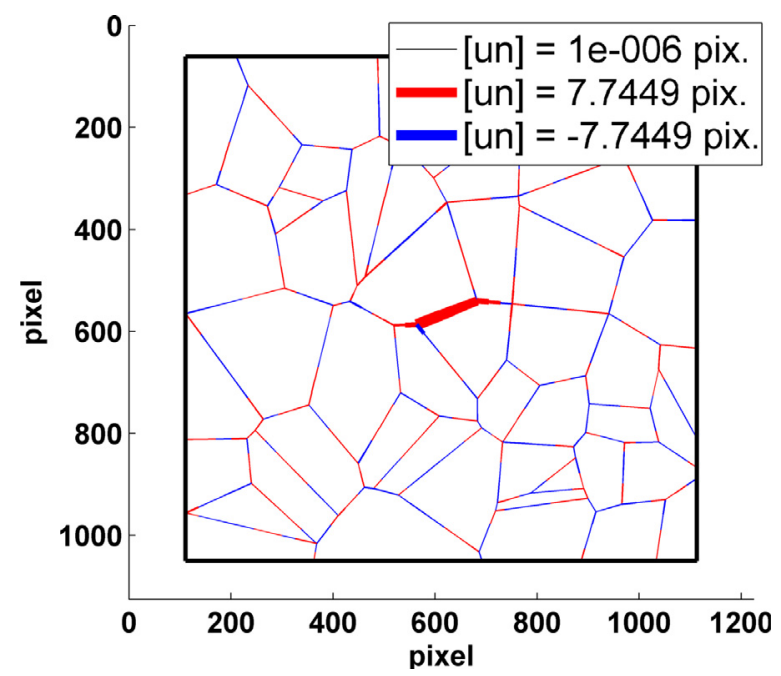

Fig. 15. Identification of the intergranular crack via the normal displacement jump.

ments. As expected, the displacement jump evolves quadratically inside each element by using the bi-linear shape functions.

With the study-III, a rigorously continuous displacement jump profile is obtained (in blue), which is even closer to the reference curve. But the trend is not exactly similar. This observation is likely the consequence of a too high number of kinematic restrictions. In fact, the continuity restriction is imposed on all the boundary points (exact continuity restriction), so there is not enough DOFs for an accurate local kinematic description.

In order to increase the number of DOFs for the kinematic description, the level of the imposed continuity restriction is decreased. Instead of imposing the continuity condition for all the points along each element boundary within each grain, a partially relaxed continuity restriction is applied. This condition consist in enforcing continuity only on the two extremes points of the boundary instead of the full boundary. The relaxed continuity allows to have more DOFs number $(=4290$ compared to 1372), resulting in a better local crack description as shown in black in Fig. 14, which is referred to as Study-III-Partial.

Using the result of the last study (with partial continuity condition), we can additionally investigate the normal displacement jump $\left(\mathbf{u}_{\mathbf{n}}\right)$ for all the grain boundaries (according theirs local Normal-Tangential coordinate system) inside of ZOI and draw all of them in the Fig. 15.

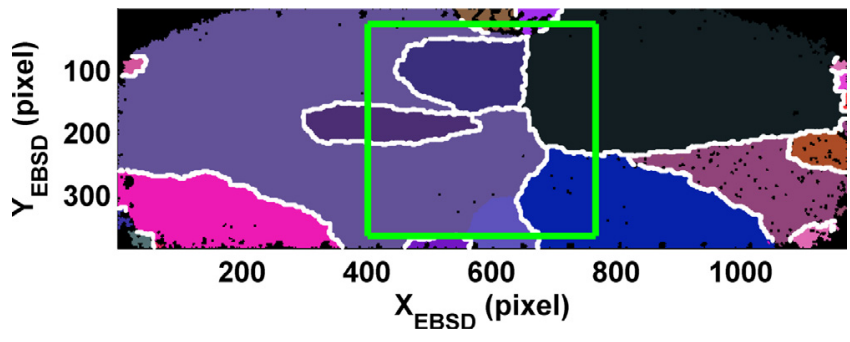

Fig. 16. Initial microstructure analysis of specimen $\left(60 \times 20 \mathrm{~mm}^{2}\right)$.

Table 1

Main camera characteristics.

\begin{tabular}{llll}
\hline & Image size (pixel) & Scale factor $(\mu \mathrm{m} /$ pixel) & Frame rate $(\mathrm{Hz})$ \\
\hline Cedip Titanium & $512 \times 640$ & 97 & 40 \\
Phantom V12 & $1280 \times 800$ & 45 & 80 \\
\hline
\end{tabular}

As expected, the displacement jump is only found in the introduced cracking zone. In the Fig. 15, the width of the segment corresponds to the amplitude of the displacement jump, and the width corresponding the maximum displacement jump is represented in the legend of figure. And the blue color is for negative jump and the red color is for positive jump. With such investigation, only the introduced intergranular crack is clearly identified and localized, so, the discontinuity capturing ability of Constrained DIC method is obviously demonstrated.

For this numerical validation section, the comparison of kinematic fields (displacement and strain) calculated by Constrained DIC and obtained by FE simulation is conclusive and validates our methodology. In the following paper, this Constrained DIC data processing method will be applied on experimental test, which can potentially be coupled with the local thermal measurement method [9] for the grain-scaled energy balance establishing.

\section{Experimental procedures and results}

\subsection{Microstructure analysis}

In this experimental study, the as-received material used consists in a $3 \mathrm{~mm}$ thick aluminium sheet, grade $A 1050$. The chemical composition of this material and the sample preparation procedure is reported in $[6]$.

The initial microstructure is analysed by EBSD before mechanical testing and the microstructural map is represented in the following figure:

Each color in the Fig. 16 corresponds to a crystal orientation (interpreted here as a grain) and the white line materializes the high disorientation zone associated with grain boundaries. The green frame in this figure corresponds to the Zone Of Interest (ZOI) defined in the CCD and IR coordinate systems (see Fig. 17) but transported in the EBSD coordinate system.

\subsection{Experimental setup}

In this study, mechanical (load-unload) tensile tests are performed at room temperature with a hydraulic testing machine (MTS-810) in a displacement-controlled mode. A simultaneous observation of both sides of the sample is performed by the CCD and IR cameras. The main characteristics of the cameras are reported in Table 1.

Thanks to a spatial matching procedure [6], the grain boundaries (white in Fig. 16) are respectively transported in the CCD and IR coordinate systems (in Fig. 17a and b).

In Fig. 17, the contour of the grain boundaries given by EBSD analysis are represented in black. In the ZOI (green frame), this microstructure is then "simplified" in order to obtain meshes with a "reasonable" size 


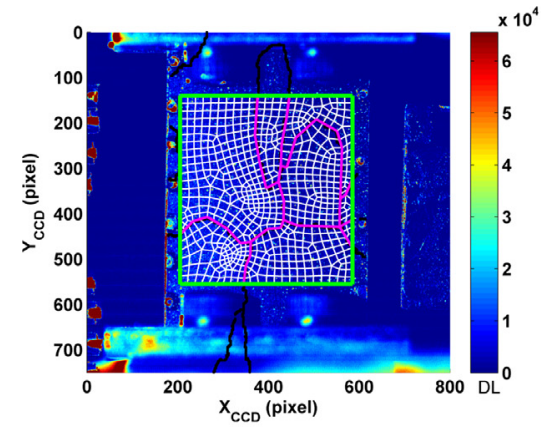

(a) Correlation analysis mesh

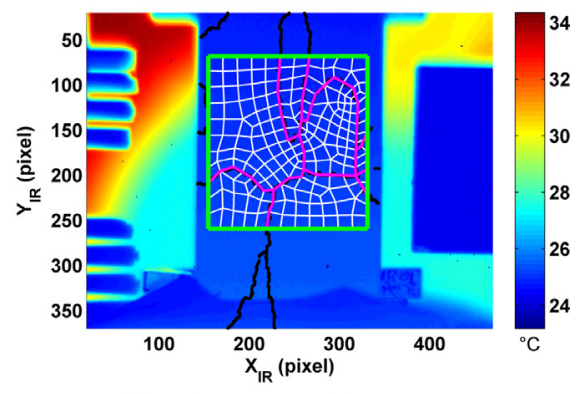

(b) Thermal analysis mesh

Fig. 17. Spatial description of the specimen in the CCD and IR coordinate systems.

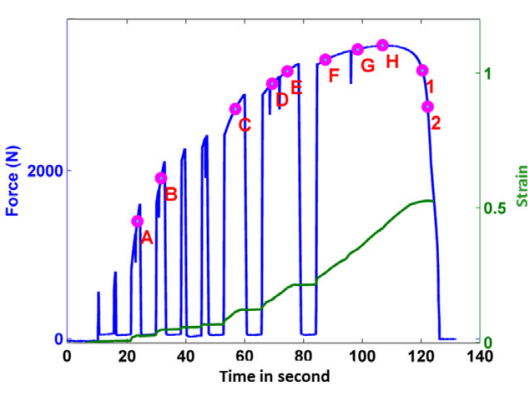

(a) Mechanical and kinematic responses

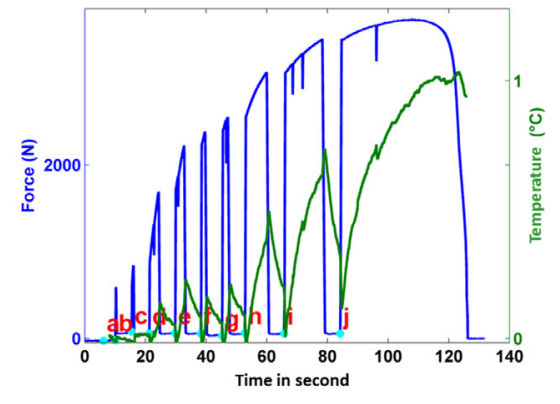

(b) Mechanical and thermal responses

Fig. 18. Macroscopic responses of material.

for the image correlation and the calorimetric analysis. According the different spatial resolution of the CCD and IR cameras (Table 1), the mesh sizes used here for the kinematic and the thermal analysis are respectively 18 pixels and 40 pixels.

\subsection{Macroscopic response}

The macroscopic response of the aluminum sample for this loadunload tensile test (in Y direction of Fig. 17) until failure is reported in Fig. 18. The mechanical loading is represented in blue, the macroscopic kinematic and thermal responses (i.e. averaged over the ZOI) are respectively represented in green in the Fig. 18a and b.

\subsection{Full field analysis}

After the macroscopic temporal evolutions, the grain-scaled result will be presented in this section. The Constrained DIC method is used in order to perform the full field analysis. In this paper, the results at the loading state specified by the D loading step (in Fig. 18a) are presented at first.

\subsubsection{Displacement fields}

As illustrated in Fig. 17a, there are 8 grains inside of ZOI. The data processing applied here involves a displacement continuity for all the adjacent elements within each grain, and no continuity relation on the grain boundaries, which is equivalent to an intragranular displacement continuity inside of each grain. A bi-linear shape function is used for the local kinematic description for each element. And the SSD criterion (Sun of Squared Differences) is used for DIC processing, as mentioned in Eq. (7).

In this stage of the post-processing, the grain-scaled displacement fields in the two directions are obtained with the Constrained DIC method. These displacement fields are represented in the Lagrangian configuration, as shown in Fig. 19. We can naturally represent the displacement fields in their Eulerian configuration.

\subsubsection{Initial and deformed measurement mesh}

Using the obtained displacement fields (in Fig. 19), the initial kinematic mesh (Fig. 17a) can be deformed to obtain the current one in the CCD coordinate system (Fig. 20a). Using the spatial matching procedure, the initial and deformed thermal mesh can be also represented in the CCD coordinate system (Fig. 20b) and then in the IRT coordinate system.

In Fig. 20, the initial and deformed computational mesh (DIC and IRT) are presented, together with the corresponding deformed visible image. Using this procedure, the microstructure evolution can be monitored in real time during the test.

\subsubsection{Equivalent Von Mises strain field}

The deformed mesh and the deformed image corresponding to the same loading step D are presented in Fig. 21, and the color of mesh (except the mesh contour in yellow) are represented the equivalent strain level in the current configuration.

This figure illustrates the heterogeneous development of plasticity between and within grains (strain localisation).

Apart from the strain localisation in different grains, a kinematic jump is clearly identified in the area surrounded in magenta (in Fig. 21). In fact, this area is corresponded the location where the initial crack is identified.

\subsubsection{Initial crack}

In Fig. 22a, the equivalent strain field for loading step C (in Fig. 18a) with the corresponding deformed image has been presented in Eulerian configuration.

The distribution of strong strain localization and the kinematic jump are clearly identified in the same area as shown in Fig. 21. In addition, kinematic jump is better highlighted in Fig. 22b, from which the initial crack can be remarked. 


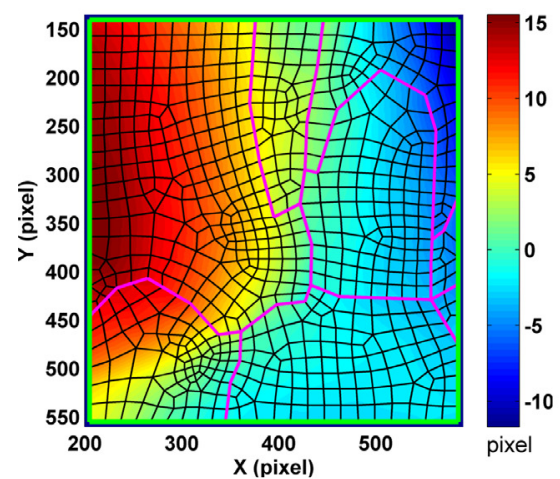

(a) Displacement field in $\mathrm{X}$ direction

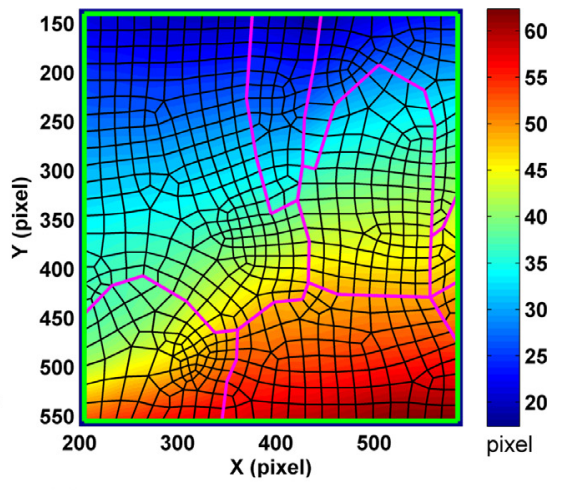

(b) Displacement field in $\mathrm{Y}$ direction

Fig. 19. Displacement fields obtained at loading step $D$ in the Lagrangian configuration.

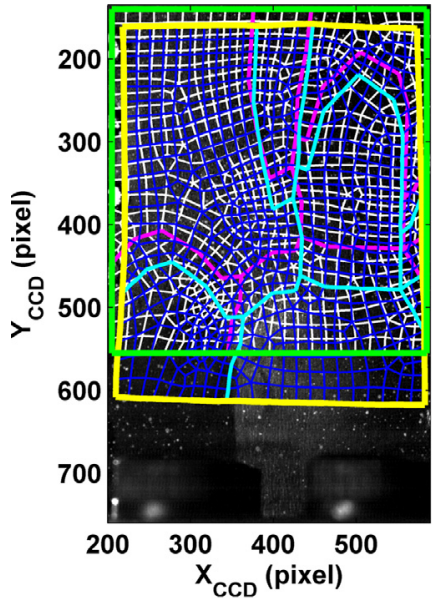

(a) Initial and deformed CCD mesh represented in the CCD coordinate system

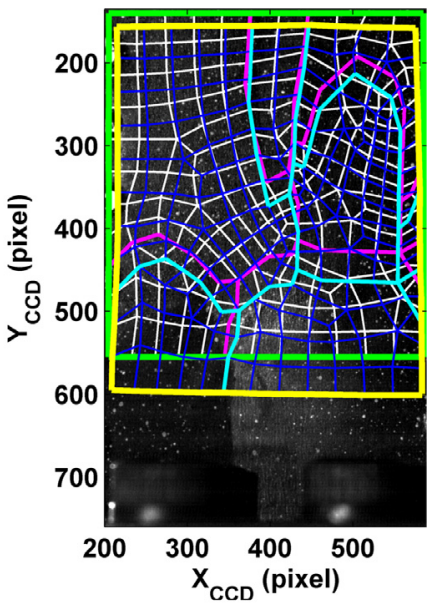

(b) Initial and deformed IRT mesh represented in the CCD coordinate system

Fig. 20. Initial and deformed mesh represented in the CCD coordinate system for loading step D.

\subsubsection{Ultimate fracture}

For the loading step 2 in Fig. 18a, the corresponding equivalent strain field with the deformed image is presented in Fig. 23a. And, the zoom of this representation is shown in Fig. 23b.

With the tracking operation of the microstructure, the initial crack can be always localized until the last stage of test, despite the coating is well degraded. This phenomena demonstrates that the displacement

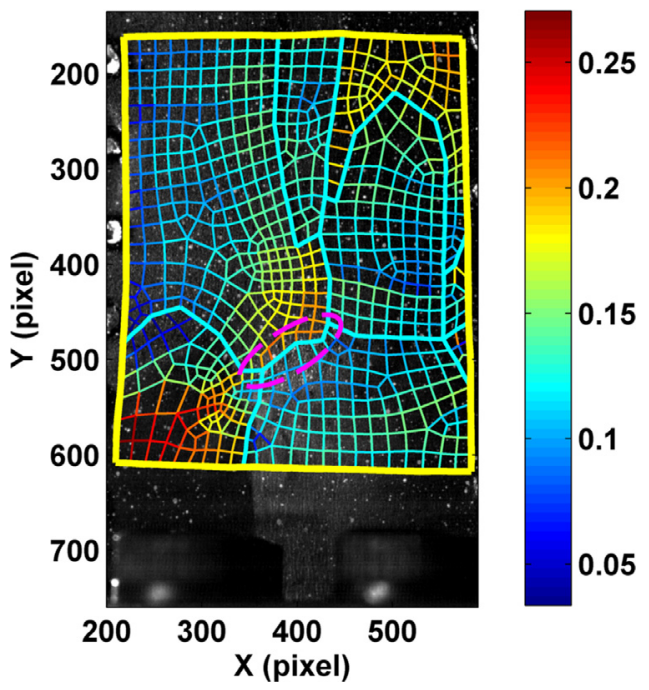

Fig. 21. Equivalent strain field in Eulerian configuration for loading step D.

fields obtained by Constrained DIC, used then for the computational mesh monitoring operation, are suitably corresponded the real deformation during the experimentation.

Simultaneously, the ultimate fracture is also clearly identified with Constrained DIC method. For a better illustration, the ultimate crack is highlighted by a white dashed line in the Fig. 23b. The ultimate fracture has occurred exactly at the most deformed locus as revealed by the

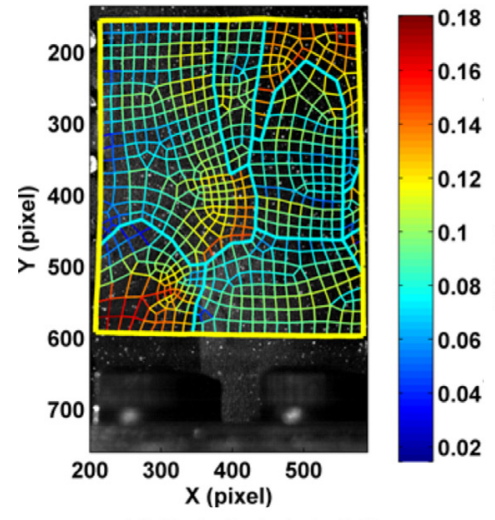

(a) Equivalent strain field

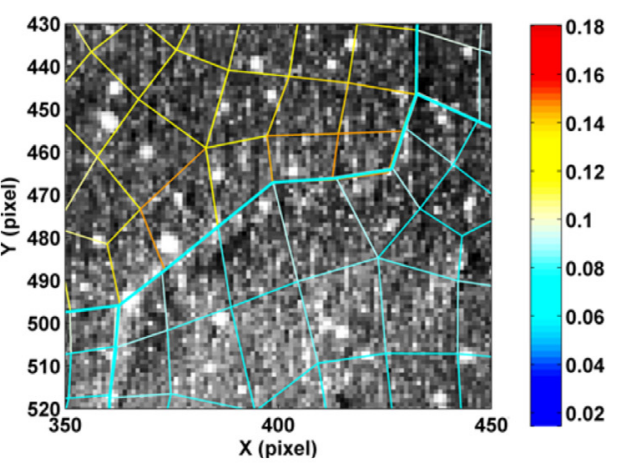

(b) Zoom of equivalent strain field

Fig. 22. Kinematic field obtained at loading step $\mathrm{C}$ in the Eulerian configuration. 


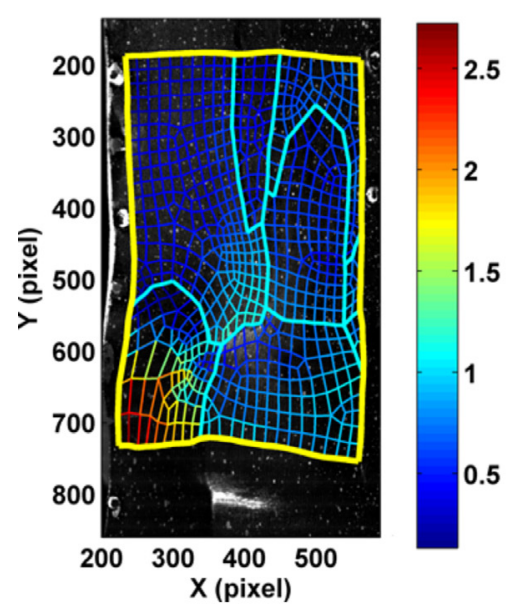

(a) Equivalent strain field

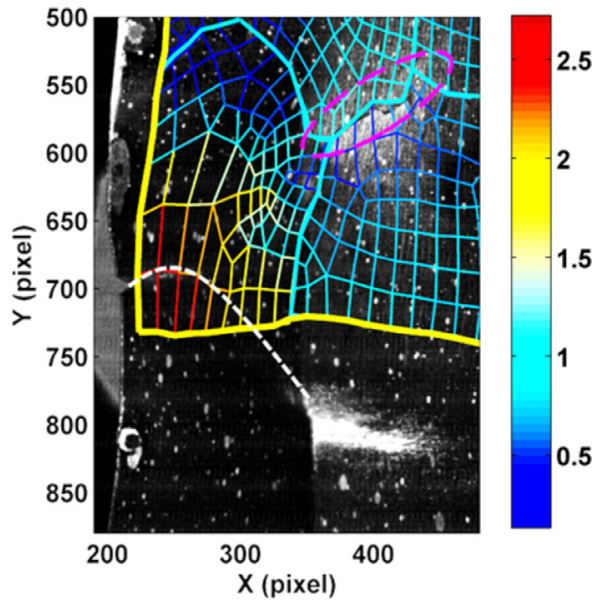

(b) Zoom of equivalent strain field

Fig. 23. Kinematic field obtained at loading step 2 in the Eulerian configuration.

Constrained DIC processing. Moreover, an incremental strain computation is used for this data processing, so a strain exceeding $100 \%$ was obtained in this final stage of experimentation.

\section{Conclusion}

Through this paper, a kinematic data processing method is proposed, which is possible to take into account directly the microstructure for the computation of the displacement field and the strain field. Using this method, the more or less complex kinematic description can be performed with the different shape functions. And, the intergranular and/or the intragranular effect can be explicitly considered in the data processing procedure, via the different type of restriction and the different level of restriction. The material effects can be explicitly introduced in the data processing procedure, while respecting the physical microstructure given by EBSD analysis, is the key feature of this proposed method, which fit the polycrystalline problem.

The validation procedure of the proposed method is performed on a numerical example, which is associated with a complex situation corresponding a cracked polycrystalline aggregates. Despite the imposed kinematic fields are very heterogeneous with strong gradient, however, for a $2 \%$ measured macroscopic strain (more than $10 \%$ locally), the obtained results show a quantitatively correct estimate with the Mean Error and the Standard Deviation being respectively in order of magnitude of $10^{-3}$ and $10^{-4}$. All of these results are conclusive to validate our methodology. Furthermore, for completely enclosing this numerical validation stage, the robustness of the proposed method with respect to superimposed noise should be investigated in more detail.

Afterwards, this proposed method was applied to a real experimental application. If we focus only in the kinematic part, a very early crack detection is shown with the obtained result. Then, we can also follow the specimen deformation until the ultimate fracture with the kinematic analysis.

In a general way, using the Constrained methods (DIC and TIR [9]), the local distributions of strain and temperature fields can be measured in the coarse-grained aluminium polycrystal. Therefore, the different local thermomechanical variables can be characterised specifically for each grain (without introducing correlations with the response of adjacent grains).

By construction, such measurements can potentially be confronted to EBSD analyses which giving the grains initial orientation, in order to estimate the local stress fields within each grain that are needed to determine the deformation energy locally developed [41]. This is the last step required for the proposition of a complete energy balance at the scale of grain.

Appendix A. Appendix : analytical example for restriction matrix construction

We propose here to illustrate this Constrained DIC method in the analytical case that the continuity restriction is imposed on the displacement vector between two elements (elements $i$ and $j$ ) in Fig. 2 with a relatively "horizontal" boundary.

Two analytical examples will be presented to introduce normal continuity and tangential continuity on the boundary $l_{u}^{i j}$ between the elements $i$ and $j$. For both cases, a bi-linear shape function is chooses for both elements $i$ and $j$ :

- Bi-linear shape function for element $i$ :

$$
\begin{aligned}
& \mathbf{u}_{\mathbf{x}}^{\mathbf{i}}\left(X_{C C D}, Y_{C C D}, p_{X}^{i}\right)=a_{00}^{i}+a_{10}^{i} X_{C C D}+a_{01}^{i} Y_{C C D}+a_{11}^{i} X_{C C D} Y_{C C D} \\
& \mathbf{u}_{\mathbf{y}}^{\mathbf{i}}\left(X_{C C D}, Y_{C C D}, p_{Y}^{i}\right)=b_{00}^{i}+b_{10}^{i} X_{C C D}+b_{01}^{i} Y_{C C D}+b_{11}^{i} X_{C C D} Y_{C C D}
\end{aligned}
$$

- Bi-linear shape function for element $j$ :

$$
\begin{aligned}
& \mathbf{u}_{\mathbf{x}}^{\mathbf{j}}\left(X_{C C D}, Y_{C C D}, p_{X}^{j}\right)=a_{00}^{j}+a_{10}^{j} X_{C C D}+a_{01}^{j} Y_{C C D}+a_{11}^{j} X_{C C D} Y_{C C D} \\
& \mathbf{u}_{\mathbf{y}}^{\mathbf{j}}\left(X_{C C D}, Y_{C C D}, p_{Y}^{j}\right)=b_{00}^{j}+b_{10}^{j} X_{C C D}+b_{01}^{j} Y_{C C D}+b_{11}^{j} X_{C C D} Y_{C C D}
\end{aligned}
$$

Hence, it is noted that the two displacement vectors of the element $i$ and $j$ are expressed as follows:

$\overrightarrow{\mathbf{u}}^{\mathbf{i}}=\left(\begin{array}{c}\mathbf{u}_{\mathbf{x}}^{\mathbf{i}} \\ \mathbf{u}_{\mathbf{y}}^{\mathbf{i}}\end{array}\right)$ and $\overrightarrow{\mathbf{u}}^{\mathbf{j}}=\left(\begin{array}{c}\mathbf{u}_{\mathbf{x}}^{\mathbf{j}} \\ \mathbf{u}_{\mathbf{y}}^{\mathbf{j}}\end{array}\right)$

For a relatively "horizontal" boundary likes in Fig. 2, by combing Eqs. (3) and (16), the continuities on displacement in the normal and tangential direction could be written:

- Normal continuity restriction of displacement

$$
\begin{gathered}
\overrightarrow{\mathbf{u}}^{\mathbf{i}} \cdot \vec{N}^{i j}=\overrightarrow{\mathbf{u}}^{\mathbf{j}} \cdot \vec{N}^{i j} \forall(X, Y) \in l_{u}^{i j} \\
\operatorname{soit}\left(\begin{array}{cc}
\mathbf{u}_{\mathbf{x}}^{\mathbf{i}} & \mathbf{u}_{\mathbf{y}}^{\mathbf{i}}
\end{array}\right) \cdot\left(\begin{array}{c}
-\alpha_{u} \\
1
\end{array}\right)-\left(\begin{array}{cc}
\mathbf{u}_{\mathbf{x}}^{\mathbf{j}} & \mathbf{u}_{\mathbf{y}}^{\mathbf{j}}
\end{array}\right) \cdot\left(\begin{array}{c}
-\alpha_{u} \\
1
\end{array}\right)=0 \\
\Longrightarrow-\alpha_{u} \mathbf{u}_{\mathbf{x}}^{\mathbf{i}}+\alpha_{u} \mathbf{u}_{\mathbf{x}}^{\mathbf{j}}+\mathbf{u}_{\mathbf{y}}^{\mathbf{i}}-\mathbf{u}_{\mathbf{y}}^{\mathbf{j}}=0
\end{gathered}
$$

- Tangential continuity restriction of displacement

$$
\begin{gathered}
\overrightarrow{\mathbf{u}}^{\mathbf{i}} \cdot \vec{T}^{i j}=\overrightarrow{\mathbf{u}}^{\mathbf{j}} \cdot \vec{T}^{i j} \forall(X, Y) \in l_{u}^{i j} \\
\operatorname{soit}\left(\begin{array}{cc}
\mathbf{u}_{\mathbf{x}}^{\mathbf{i}} & \mathbf{u}_{\mathbf{y}}^{\mathbf{i}}
\end{array}\right) \cdot\left(\begin{array}{c}
1 \\
\alpha_{u}
\end{array}\right)-\left(\begin{array}{cc}
\mathbf{u}_{\mathbf{x}}^{\mathbf{j}} & \mathbf{u}_{\mathbf{y}}^{\mathbf{j}}
\end{array}\right) \cdot\left(\begin{array}{c}
1 \\
\alpha_{u}
\end{array}\right)=0 \\
\Longrightarrow \mathbf{u}_{\mathbf{x}}^{\mathbf{i}}-\mathbf{u}_{\mathbf{x}}^{\mathbf{j}}+\alpha_{u} \mathbf{u}_{\mathbf{y}}^{\mathbf{i}}-\alpha_{u} \mathbf{u}_{\mathbf{y}}^{\mathbf{j}}=0
\end{gathered}
$$




\section{- Introduction of the Normal continuity restriction on displacement}

In order to take into account a normal continuity restriction on displacement on the relatively "horizontal" boundary $l_{u}^{i j}$ likes in Fig. 2 , we introduce the Eqs. (14) and (15) in the expression of the restricton (17):

$$
\begin{aligned}
& \left\{-\alpha_{u}^{2} a_{11}^{i}+\alpha_{u}^{2} a_{11}^{j}+\alpha_{u} b_{11}^{i}-\alpha_{u} b_{11}^{j}\right\} X_{C C D}^{2}+\left\{\left(-\alpha_{u} a_{10}^{i}+\alpha_{u} a_{10}^{j}+b_{10}^{i}-b_{10}^{j}\right)+\left(-\alpha_{u}^{2} a_{01}^{i}+\alpha_{u}^{2} a_{01}^{j}+\alpha_{u} b_{01}^{i}-\alpha_{u} b_{01}^{j}\right)\right. \\
& \left.+\left(-\alpha_{u} \beta_{u} a_{11}^{i}+\alpha_{u} \beta_{u} a_{11}^{j}+\beta_{u} b_{11}^{i}-\beta_{u} b_{11}^{j}\right)\right\} X_{C C D}+\left\{\left(-\alpha_{u} a_{00}^{i}+\alpha_{u} a_{00}^{j}+b_{00}^{i}-b_{00}^{j}\right)+\left(-\alpha_{u} \beta_{u} a_{01}^{i}+\alpha_{u} \beta_{u} a_{01}^{j}+\beta_{u} b_{01}^{i}-\beta_{u} b_{01}^{j}\right)\right\}=0
\end{aligned}
$$

Thus, we obtain a polynomial expression of degree 2 in $X_{C C D}$. For this condition to be respected, each coefficent of the monomials of $X_{C C D}$ must be equal to zero, which gives 3 linear equations with respect to the unknown parameters $p^{i}\left(a_{00}^{i}, a_{10}^{i}, a_{01}^{i}, a_{11}^{i}, b_{00}^{i}, b_{10}^{i}, b_{01}^{i}, b_{11}^{i}\right)$ and $p^{j}\left(a_{00}^{j}, a_{10}^{j}, a_{01}^{j}, a_{11}^{j}, b_{00}^{j}, b_{10}^{j}, b_{01}^{j}, b_{11}^{j}\right)$. These linear equations could be presented in matrix form as follow:

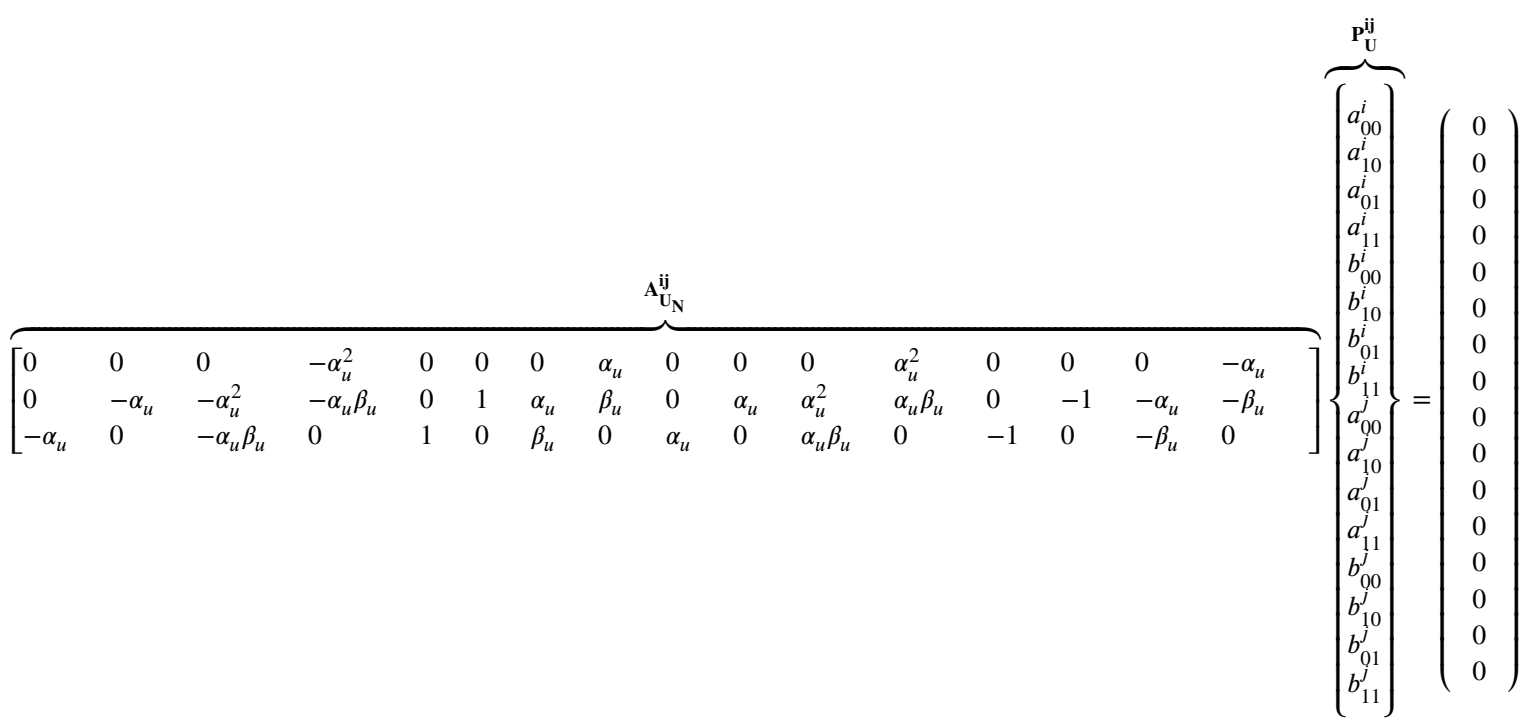

Note that a similar system can be obtained if the continuity is enforced "only" on a set of points on the boundary $l_{u}^{i j}$.

- Introduction of the Tangential continuity restriction on displacement

In the same way, taking into account of the tangential continuity restriction on displacement on boundary $l_{u}^{i j}$ gives:

$$
\begin{aligned}
& \left\{\alpha_{u} a_{11}^{i}-\alpha_{u} a_{11}^{j}+\alpha_{u}^{2} b_{11}^{i}-\alpha_{u}^{2} b_{11}^{j}\right\} X_{C C D}^{2}+\left\{\left(a_{10}^{i}-a_{10}^{j}+\alpha_{u} b_{10}^{i}-\alpha_{u} b_{10}^{j}\right)+\left(\alpha_{u} a_{01}^{i}-\alpha_{u} a_{01}^{j}+\alpha_{u}^{2} b_{01}^{i}-\alpha_{u}^{2} b_{01}^{j}\right)\right. \\
& \left.+\left(\beta_{u} a_{11}^{i}-\beta_{u} a_{11}^{j}+\alpha_{u} \beta_{u} b_{11}^{i}-\alpha_{u} \beta_{u} b_{11}^{j}\right)\right\} X_{C C D}+\left\{\left(a_{00}^{i}-a_{00}^{j}+\alpha_{u} b_{00}^{i}-\alpha_{u} b_{00}^{j}\right)+\left(\beta_{u} a_{01}^{i}-\beta_{u} a_{01}^{j}+\alpha_{u} \beta_{u} b_{01}^{i}-\alpha_{u} \beta_{u} b_{01}^{j}\right)\right\}=0
\end{aligned}
$$

This system is written in matrix form as follow:

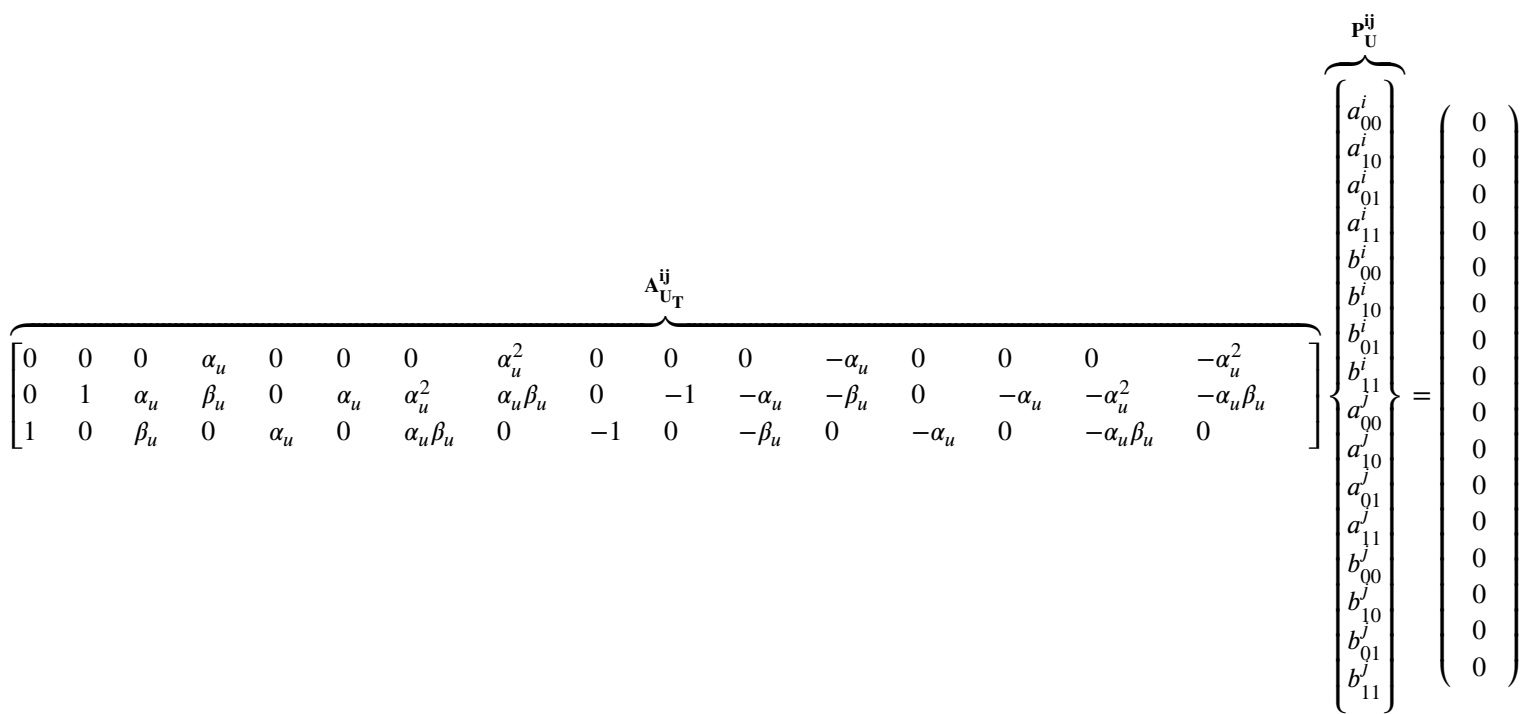

The two systems (Eqs. (19) and (20)) correspond to the separate introduction of normal and tangential continuities on the boundary. It is possible to simultaneously introduce these two continuities on the same boundary, by assembling the normal restriction matrix (A $\mathbf{A}_{\mathbf{N}}^{\mathrm{ij}}$ ) and the tangential restriction matrix $\left(\mathbf{A}_{\mathbf{U}_{\mathbf{T}}}^{\mathrm{ij}}\right.$ ) to construct the normal-tangential restriction matrix $\mathbf{A}_{\mathbf{U}}^{\mathrm{ij}}$ which takes into account these two types of continuity at the same 


\begin{tabular}{|c|c|c|c|}
\hline$[0$ & 0 & 0 & $-\alpha_{u}^{2}$ \\
\hline 0 & $-\alpha_{u}$ & $-\alpha_{u}^{2}$ & $-\alpha_{u} \beta_{u}$ \\
\hline$-\alpha_{u}$ & 0 & $-\alpha_{u} \beta_{u}$ & 0 \\
\hline 0 & 0 & 0 & $\alpha_{u}$ \\
\hline 0 & 1 & $\alpha_{u}$ & $\beta_{u}$ \\
\hline L1 & 0 & $\beta_{u}$ & 0 \\
\hline
\end{tabular}

$\begin{array}{lll}0 & 0 & 0 \\ 0 & 1 & \alpha_{u} \\ 1 & 0 & \beta_{u} \\ 0 & 0 & 0 \\ 0 & \alpha_{u} & \alpha_{u}^{2} \\ \alpha_{u} & 0 & \alpha_{u} \beta_{u}\end{array}$

$\alpha_{u}$
$\beta_{u}$
0
$\alpha_{u}^{2}$
$\alpha_{u} \beta_{u}$
0

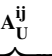

$\left.\begin{array}{llllllll}0 & 0 & 0 & \alpha_{u}^{2} & 0 & 0 & 0 & -\alpha_{u} \\ 0 & \alpha_{u} & \alpha_{u}^{2} & \alpha_{u} \beta_{u} & 0 & -1 & -\alpha_{u} & -\beta_{u} \\ \alpha_{u} & 0 & \alpha_{u} \beta_{u} & 0 & -1 & 0 & -\beta_{u} & 0 \\ 0 & 0 & 0 & -\alpha_{u} & 0 & 0 & 0 & -\alpha_{u}^{2} \\ 0 & -1 & -\alpha_{u} & -\beta_{u} & 0 & -\alpha_{u} & -\alpha_{u}^{2} & -\alpha_{u} \beta_{u} \\ -1 & 0 & -\beta_{u} & 0 & -\alpha_{u} & 0 & -\alpha_{u} \beta_{u} & 0\end{array}\right]$

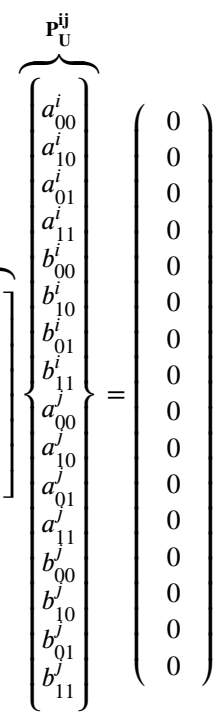

Like in Eq. (5), where $\left[\mathbf{A}_{\mathbf{U}}^{\mathbf{i j}}\right]$ is the kinematic elementary restriction matrix between element $i$ and $j$, and $\left\{\mathbf{P}_{\mathbf{U}}^{\mathbf{i j}}\right\}$ is the elementary vector containing all the unknown kinematic parameters $\left(\mathbf{p}^{\mathbf{i}}, \mathbf{p}^{\mathbf{j}}\right)=\left(a_{k l}^{i}, b_{k l}^{i}, a_{k l}^{j}, b_{k l}^{j}\right)$ for these two adjacent elements $i$ and $j$.

By iterating this operation for all boundaries on which continuity restrictions are applied, a global kinematic restriction matrix $\mathbf{A}_{\mathbf{U}}$ is built for the mesh, as well as a global vector $\mathbf{P}_{\mathbf{U}}$ containing all the kinematic parameters, like in Eq. (6).

\section{Supplementary material}

Supplementary material associated with this article can be found, in the online version, at 10.1016/j.optlaseng.2018.08.003

\section{References}

[1] Zhao Z, Ramesh M, Raabe D, Cuitino A, Radovitzky R. Investigation of threedimensional aspects of grain-scale plastic surface deformation of an aluminum oligocrystal. Int J Plast 2008;24(12):2278-97. doi:10.1016/j.ijplas.2008.01.002. URL http://www.sciencedirect.com/science/article/pii/S074964190800020X

[2] Saai A, Louche H, Tabourot L, Chang H. Experimental and numerical study of the thermo-mechanical behavior of al bi-crystal in tension using full field measurements and micromechanical modeling. Mech Mater 2010;42(3):275-92. doi:10.1016/j.mechmat.2009.11.011. URL http://www.sciencedirect.com/science/article/pii/S0167663609001987

[3] Badulescu C, Grédiac M, Haddadi H, Mathias J-D, Balandraud X, Tran H-S. Applying the grid method and infrared thermography to investigate plastic deformation in aluminium multicrystal. Mech Mater 2011;43(1):36-53. doi:10.1016/j.mechmat.2010.11.001. URL http://www.sciencedirect.com/science/article/pii/S0167663610001535

[4] Bodelot L, Charkaluk E, Sabatier L, Dufrénoy P. Experimental study of heterogeneities in strain and temperature fields at the microstructural level of polycrystalline metals through fully-coupled full-field measurements by digital image correlation and infrared thermography. Mech Mater 2011;43(11):654-70. doi:10.1016/j.mechmat.2011.08.006. URL http://www.sciencedirect.com/science/article/pii/S0167663611001554

[5] Seghir R, Witz J-F, Bodelot L, Charkaluk E, DufrEnoy P. An improved lagrangian thermography procedure for the quantification of the temperature fields within polycrystals. Quant Infrared Thermogr J 2013;10(1):74-95. doi:10.1080/17686733.2013.785207. URL https://doi.org/10.1080/17686733.2013.785207

[6] Li L, Muracciole J-M, Sabatier L, Waltz L, Wattrisse B. Analysis of the thermo-mechanical behavior of coarse-grained polycrystalline aluminum under tensile conditions. PhotoMechanics 2013. Montpellier, France; 2013. URL http://hal.archives-ouvertes.fr/hal-00836313

[7] Li L, Latourte F, Muracciole JM, Waltz L, Sabatier L, Wattrisse B. Capturing polycrystal plasticity and intergranular cracks with a novel DIC method. 11th World Congress on Computational Mechanics (WCCM XI). Barcelone, Spain; 2014. URL https://hal.archives-ouvertes.fr/hal-01175898

[8] Li L. Caractérisation et identification du comportement thermomécanique de multi-cristaux d'aluminium. Montpellier University; 2014.

[9] Li L, Latourte F, Muracciole J-M, Waltz L, Sabatier L, Wattrisse B. Calorimetric analysis of coarse-grained polycrystalline aluminum by irt and dic. Quant Infrared Thermogr J 2015;12(01):80-97. doi:10.1080/17686733.2015.1022354. URL http://www.tandfonline.com/doi/abs/10.1080/17686733.2015.1022354
[10] Sutton M, McNeill S, Helm J, Chao Y. Advances in two-dimensional and three-dimensional computer vision. In: Rastogi P, editor. Photomechanics. Topics in Applied Physics, 77. Springer Berlin Heidelberg; 2000. p. 323-72. ISBN 978-3-540-65990-7. doi:10.1007/3-540-48800-6_10. URL https://doi.org/10.1007/3-540-48800-6_10

[11] Sutton M, Wolters W, Peters W, Ranson W, McNeill S. Determination of displacements using an improved digital correlation method. Image Vis Comput 1983;1(3):133-9. https://doi.org/10.1016/0262-8856(83)90064-1. URL http://www.sciencedirect.com/science/article/pii/0262885683900641

[12] Wattrisse B, Chrysochoos A, Muracciole J-M, Nemoz-Gaillard M. Analysis of strain localization during tensile tests by digital image correlation. Exp Mech 2001;41:29 39. doi:10.1007/BF02323101. URL https://doi.org/10.1007/BF02323101

[13] Hild F, Roux S. Digital image correlation: from displacement measurement to identification of elastic properties: a review. Strain 2006;42(2):69-80. doi:10.1111/j.1475-1305.2006.00258.x. https://doi.org/10.1111/j.1475-1305.2006.00258.x

[14] Pan B, Qian K, Xie H, Asundi A. Two-dimensional digital image correlation for in-plane displacement and strain measurement: a review. Meas Sci Technol 2009;20(6):062001. URL http://stacks.iop.org/0957-0233/20/i=6/a=062001

[15] Boas W, Hargreaves ME. On the inhomogeneity of plastic deformation in the crystals of an aggregate. In: Proceedings of the royal society of london series a mathematical and physical sciences, 193; 1948. p. 89-97. doi:10.1098/rspa.1948.0035. http://rspa.royalsocietypublishing.org/content/193/1032/89.abstract

[16] Jaoul B. Étude de la plasticité et application aux métaux. Dunod; 1965. URL http://books.google.fr/books?id=EuLAygAACAAJ

[17] Raabe D, Sachtleber M, Zhao Z, Roters F, Zaefferer S. Micromechanical and macromechanical effects in grain scale polycrystal plasticity experimentation and simulation. Acta Mater 2001;49(17):3433-41. doi:10.1016/S1359-6454(01)00242-7. http://www.sciencedirect.com/science/article/pii/S1359645401002427

[18] Sachtleber M, Zhao Z, Raabe D. Experimental investigation of plastic grain interaction. Mater Sci Eng 2002;336(12):81-7. https://doi.org/10.1016/S0921-5093(01)01974-8. URL http://www.sciencedirect.com/science/article/pii/S0921509301019748

[19] Héripré E, Dexet M, Crépin J, Gélébart L, Roos A, Bornert M, et al. Coupling between experimental measurements and polycrystal finite element calculations for micromechanical study of metallic materials. Int $\mathrm{J}$ Plast 2007;23(9):1512-39. https://doi.org/10.1016/j.ijplas.2007.01.009. URL http://www.sciencedirect.com/science/article/pii/S074964190700006X

[20] Bodelot L, Sabatier L, Charkaluk E, Dufrénoy P. Experimental setup for fully coupled kinematic and thermal measurements at the microstructure scale of an aisi 3161 steel. Mater Sci Eng 2009;501(12):52-60. doi:10.1016/j.msea.2008.09.053. URL http://www.sciencedirect.com/science/article/pii/S0921509308011167

[21] Besnard G, Hild F, Roux S. ǣFinite-elementg displacement fields analysis from digital images: application to portevinle chtelier bands. Exp Mech 2006;46(6):789-803. doi:10.1007/s11340-006-9824-8. URL https://doi.org/10.1007/s11340-006-9824-8

[22] Leclerc H, PȨriȨ J-N, Roux S, Hild F. Integrated digital image correlation for the identification of mechanical properties. In: Gagalowicz A, Philips W, 
editors. Computer Vision/Computer Graphics CollaborationTechniques. Lecture Notes in Computer Science, 5496. Springer Berlin Heidelberg; 2009. p. 161-71. ISBN 978-3-642-01810-7. doi:10.1007/978-3-642-01811-4_15. URL https://doi.org/10.1007/978-3-642-01811-4_15

[23] Cheng P, Sutton M, Schreier H, McNeill S. Full-field speckle pattern image correlation with b-spline deformation function. Exp Mech 2002;42(3):344-52. doi:10.1007/BF02410992. URL https://doi.org/10.1007/BF02410992

[24] Hild F, Roux S, Gras R, Guerrero N, Marante ME, Flrez-Lpez J. Displacement measurement technique for beam kinematics. Opt Lasers Eng 2009;47(34):495-503. https://doi.org/10.1016/j.optlaseng.2008.03.006. Optical Measurements

[25] Réthoré J, Hild F, Roux S. Extended digital image correlation with crack shape optimization. Int J Numer Methods Eng 2008;73(2):248-72. doi:10.1002/nme.2070. URL https://doi.org/10.1002/nme.2070

[26] Chen J, Zhang X, Zhan N. Extended digital image correlation method for microregion deformation measurement. Sci China Technol Sci 2011;54(6):1355-61. doi:10.1007/s11431-011-4404-2.

[27] Fennema CL, Thompson WB. Velocity determination in scenes containing several moving objects. Comput Graph Image Process 1979;9(4):301-15. https://doi.org/10.1016/0146-664X(79)90097-2. URL http://www.sciencedirect.com/science/article/pii/0146664X79900972

[28] Adams BL, Wright SI, Kunze K. Orientation imaging: the emergence of a new microscopy. Metall Trans A 1993;24:819-31. doi:10.1007/BF02656503. URL https://doi.org/10.1007/BF02656503

[29] Humphreys F. Review grain and subgrain characterisation by electron backscatter diffraction. J Mater Sci 2001;36:3833-54. doi:10.1023/A:1017973432592. URL https://doi.org/10.1023/A:3A1017973432592

[30] Menouillard T, Rethore J, Combescure A, Bung H. Efficient explicit time stepping for the extended finite element method ( $\mathrm{x}$-fem). Int $\mathrm{J}$ Numer Methods Eng 2006;68(9):911-39.

[31] Hild F, Roux S. Comparison of local and global approaches to digital image correlation. Exp Mech 2012;52(9):1503-19. doi:10.1007/s11340-012-9603-7. URL https://doi.org/10.1007/s11340-012-9603-7

[32] Bornert M, Brémand F, Doumalin P, Dupré J-C, Fazzini M, Grédiac M, et al. Assessment of digital image correlation measurement errors: methodology and results. Exp Mech 2009;49:353-70. 10.1007/s11340-008-9204-7
[33] Cuitino AM, Ortiz M. Computational modelling of single crystals. Modell Simul Mater Sci Eng 1993;1(3):225. URL http://stacks.iop.org/0965-0393/1/i=3/a=001

[34] Meric L, Poubanne P, Cailletaud G. Single crystal modeling for structural calculations. i, model presentation. J Mech Design (1990) 1991;113(1):162-70. Eng; URI http://www.refdoc.fr/Detailnotice?idarticle $=19348041$

[35] Green PJ, Sibson R. Computing dirichlet tessellations in the plane. Comput J 1978;21(2):168-73. doi:10.1093/comjnl/21.2.168. URL http://comjnl.oxfordjournals.org/content/21/2/168.abstract

[36] Barbe F, Decker L, Jeulin D, Cailletaud G. Intergranular and intragranular behavior of polycrystalline aggregates. part 1: f.e. model. Int J Plast 2001;17(4):513-36. https://doi.org/10.1016/S0749-6419(00)00061-9. URL http://www.sciencedirect.com/science/article/pii/S0749641900000619

[37] Barbe F, Forest S, Cailletaud G. Intergranular and intragranular behavior of polycrystalline aggregates.part 2: results. Int $\mathrm{J}$ Plast 2001;17(4):537-63. https://doi.org/10.1016/S0749-6419(00)00062-0. URL http://www.sciencedirect.com/science/article/pii/S0749641900000620

[38] Cizelj L, Riesch-Oppermann H. Modelling the early development of secondary side stress corrosion cracks in steam generator tubes using incomplete random tessellation. Nucl Eng Des 2002;212(13):21-9. https://doi.org/10.1016/S0029-5493(01)00474-5. http://www.sciencedirect.com/science/article/pii/S0029549301004745

[39] Latourte F, Rupin N, Proix J-M. Plasticité cristalline dans un acier bainitique revenu: simulations pour la validation de modèles à; partir de mesures de champs. 11e Colloque National en Calcul des Structures, France; 2013.

[40] Wang ZY, Li HQ, Tong JW, Ruan JT. Statistical analysis of the effect of intensity pattern noise on the displacement measurement precision of digital image correlation using self-correlated images. Exp Mech 2007;47(5):701-7. doi:10.1007/s11340-006-9005-9.

[41] Madani T, Monerie Y, Pagano S, Pelissou C, Wattrisse B. Identification of heterogeneous elastoplastic behaviors using dic measurement. Photomechanics 2015; 2015. 